\title{
Ignition of uniform droplet-laden weakly turbulent flows following a laser spark
}

\author{
Pedro M. de Oliveira, Patton M. Allison, Epaminondas Mastorakos \\ Hopkinson Laboratory, Department of Engineering, University of Cambridge, \\ Cambridge, CB2 1PZ, UK
}

\begin{abstract}
The forced ignition process has a stochastic nature, which can be intensified due to turbulence and mixture fluctuations. Although fuel droplets represent strong inhomogeneities which are generally detrimental to ignition, the presence of small droplets has been found to enhance flame speeds, decrease minimum ignition energy, and improve the ignitability of overall lean mixtures. In order to understand which factors are conducive to ignition of sprays, a spherically expanding flame is investigated, which is produced by a laser spark in a uniform dispersion of ethanol droplets in turbulent air. The flame is visualised by schlieren and $\mathrm{OH}^{*}$-chemiluminescence for overall equivalence ratios of 0.8 to 2 , Sauter mean diameter of approximately $25 \mu \mathrm{m}$, and $u^{\prime} / S_{L}$ ranging from 0.9 to 1.3 , where $u^{\prime}$ and $S_{L}$ denote the rms axial velocity and laminar burning velocity, respectively. The timescales of the spark's effects on the flame are measured, as well as quenching timescales and initial kernel sizes conditional on ignition or failure. Small kernels quenched faster than approximately $0.6 \mathrm{~ms}$, that is, the duration of the flame overdrive, and a minimum kernel radius for ignition of $1 \mathrm{~mm}$ was observed. The short-mode of ignition failure was suppressed by increasing the laser energy and, consequently, the initial kernel size. Nevertheless, the ignitability of lean mixtures was only effectively improved through high-energy sparks and partial prevaporisation of the fuel. Virtually all kernels ignited once prevaporisation was increased, and the gas-phase equivalence ratio was approximately $75 \%$ of the lower flammability limit, with ignition being limited only by laser breakdown.
\end{abstract}

Keywords:

Email address: pm580@cam.ac.uk (Pedro M. de Oliveira) 
spray combustion, ignition randomness, laser ignition, ethanol

\section{Introduction}

The issue of initiation of a flame in a two-phase flow is of fundamental importance for equipment operating with liquid fuels such as aircraft gas turbines, in which high-altitude relight of the engine is a key factor determining the operation range of the aircraft. The factors preventing a flame kernel from igniting in a combustor filled with an overall flammable mixture are associated with fluctuations at the spark location, giving rise to fuel starvation by the flame and heat transfer to the surrounding flow [1]. Such fluctuations include turbulence [2], fuel concentration fluctuations [3], and those arising from the randomness of the breakdown of the mixture caused by the spark [4]. Additionally, fuel inhomogeneities are intrinsic to spray flows, and further fluctuations may occur due to droplet breakup, atomisation and evaporation processes [5]. Hence, the identification of the conditions conducive to the initiation and growth of a flame in a spray can be challenging as such fluctuations add up and interact, giving the ignition process its stochastic character. Moreover, a better understanding of the stochasticity itself would be beneficial.

Typically, the ignitability of a flow or mixture in terms of its global parameters is assessed by measuring the minimum ignition energy (MIE). In order to take into account the randomness of the process, MIE has been commonly evaluated as the energy that results in an ignition probability of 50\% [6] with ignition not necessarily defined in the exact same way among authors. In spray flows, experiments have shown that the presence of droplets at the spark location can facilitate breakdown of the mixture by a laser spark [7], increasing the probability of breakdown. Nevertheless, their presence in the kernel or its vicinity has been shown to be detrimental to ignition in cases of large or slowly-evaporating droplets at the flame front [8], and beneficial to ignition in experiments with small droplets $[9,6]$. Experiments focusing on ignition probability have only looked at the burner-scale aspects of the problem [10-12], and although some experiments have evaluated MIE in terms of global parameters of the flow [13-15], ignition probabilities have not been investigated in canonical configurations in order to understand the effects of global flow parameters and their fluctuations controlling the early-phase ignition of sprays. 
Direct numerical simulations (DNS) have provided a great deal of insight on the phenomena occurring at the droplet or spark scale during ignition. Following the deposition of energy by the spark, quenching of the flame is characterised by two distinct modes of quenching related to their timescale: short mode and long mode of ignition failure, as defined by Mastorakos [16], which can be evaluated in respect to a reference timescale such as the overdrive effect of the spray flame caused by the spark. Short-mode of failure has been observed in simulations where quenching of the flame occurred due to a low temperature of the kernel [8], due to excessive removal of heat from the kernel through stretch by intense turbulence [2], or even due to a smallsized kernel formed after energy deposition in a locally lean mixture [17]. In contrast, slowly-evaporating droplets leading to excessive heat loss and fuel starvation were characteristic of long-mode failure [17]. Typical ignition failure timescales verified in DNS and defined in terms of the chemical time scale of the flame, $t_{f}$, were approximately $0.6 t_{f}$ for short-mode failure $[2,8]$ and $1.4 t_{f}$ for long-mode failure [8]. These timescales of quenching were assessed as the time interval for the temperature of the kernel to drop from its maximum value (at the moment of spark deactivation) down to the temperature of the fresh mixture. In experiments with sprays, the flame overdrive and ignition failure timescales, as well as minimum size for ignition, have not yet been measured.

This work focuses on the characterisation of the early phase of ignition processes of an spherically expanding flame in an ethanol-air spray. Experiments are performed in a uniformly distributed dispersion of ethanol droplets in turbulent air, allowing for the ignition and visualisation of the flame in the absence of large-scale inhomogeneities of velocity and droplet number density. Measurements of incident and absorbed energy are performed in each spark event, and the flame is visualised using simultaneous high-speed schlieren and $\mathrm{OH}^{*}$-chemiluminescence imaging. In order to verify the issues raised in recent DNS works, an evaluation of the timescale of the effects of spark on the flame is presented, as well as an evaluation of the timescales of ignition failure. These timescales are investigated in terms of the effect of fuel prevaporisation, ignition energy, equivalence ratio and initial kernel size, as an attempt to identify the conditions most conducive to ignition. Additionally, probabilities of ignition as well as MIE of ethanol sprays are assessed for laser ignition. A precise characterisation of a polydispserse spray is also given, allowing for an accurate representation of the spray in future simulations of the present experiment. 


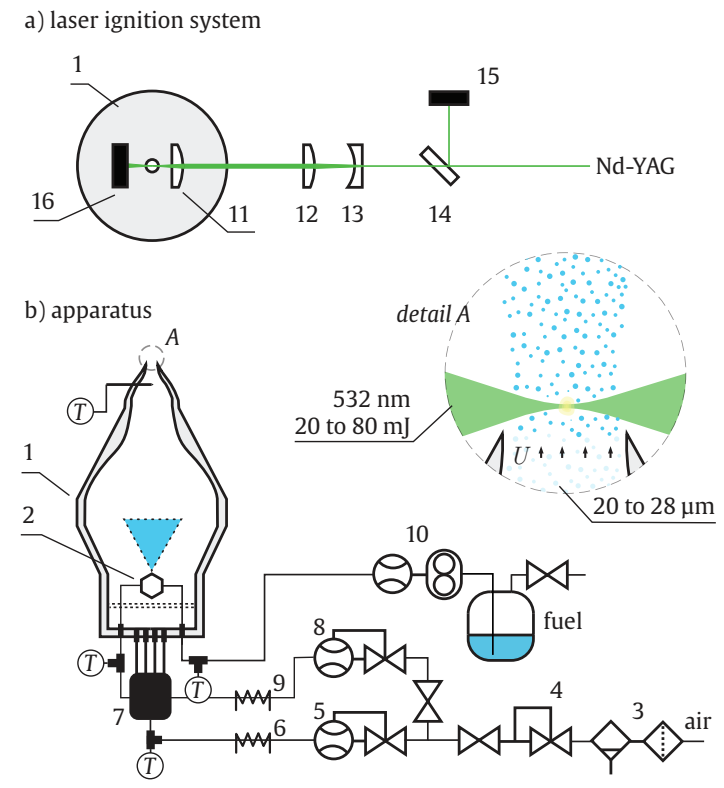

Figure 1: Schematic representation of the (a) the laser ignition system and (a) apparatus. The components of the system are: (1) burner, (2) air-assist atomiser, (3) particulate and coalescent filters, (4) pressure regulator, $(5,8)$ flow controller, $(6,9)$ in-line heater, $(7)$ flow splitter, (10) Coriolis flow meter and gear pump, (11) 30-mm plano-convex lens, (12) 75-mm plano-convex lens, (13) -30-mm plano-concave lens, (14) beam-splitter, $(15,16)$ pyroelectric energy meters.

\section{Experimental work}

\subsection{Experimental setup}

\subsubsection{Burner}

The experimental apparatus used in this work is shown schematically in Fig. 1. It consists of three fluid lines connected to a divergent-convergent tube, i.e. the burner (Fig. 1, item 1), where the two-phase fuel-air flow is formed. Inside the burner - a modified version of [18] - the liquid fuel (ethanol) is atomised by a air-assist atomiser (item 2, Delavan AL-06) in the centre of the burner. Preheated air is injected from multiple holes at the bottom of the burner. After passing through a pair of fine stainless-steel meshes, it carries the fuel droplets formed at the atomiser downstream. The mixture of fuel vapour, liquid droplets, and air, exits the burner through a 20.8-mm diameter nozzle, forming a jet. The local droplet velocity and size distribution are measured with a PDA system, as described in Sec. 2.1.3. 
Air is obtained from a compressed-air system, filtered with particulate and coalescent filters (3), and the pressure is set with regulator (4). The carrier air flow is set using a flow controller and an in-line heater with a PID temperature controller $(5,6)$, and a flow splitter is used to evenly distribute the air flow at the bottom of the burner. Similarly, the atomising-air line comprises a flow controller and a temperature-controlled in-line heater $(8,9)$. The preheating air temperature is measured in the burner, upstream of the atomiser. The liquid fuel is stored in a small reservoir at ambient temperature, and pumped into the air-assist atomiser by a gear pump controlled by a Coriolis mass flow meter (10). The global equivalence ratio of the flow results from the air and fuel mass flow rates which are independently set for each test condition.

\subsubsection{Laser ignition}

The jet is ignited with a Nd-YAG laser (Continuum Surelite II) beam at $5 \mathrm{~mm}$ downstream of the nozzle (illustrated in Fig. 1, detail A). A 532$\mathrm{nm}$ laser beam is used, with diameter of $5 \mathrm{~mm}$ and pulse duration of 4-6 ns. The laser ignition system consists of a 30-mm plano-convex lens (Fig. 1, item 11) to focus the beam, and a $75-\mathrm{mm}$ plano-convex lens and a $-30-\mathrm{mm}$ plano-concave lens $(12,13)$ to expand the beam before the focusing lens. This ignition configuration leads to breakdown of the mixture consistently at the same point at the centre of the jet. The combination of lenses resulted in a beam with a $3-\mu \mathrm{m}$ waist at its narrowest point, calculated similarly to [19], and Rayleigh length of $53 \mu \mathrm{m}$, approximately.

The dimensions of the focusing volume are of the same order as the droplet size $(5-80 \mu \mathrm{m})$, smaller than the average inter-droplet distance of the flow $(300-500 \mu \mathrm{m})$, and also smaller than the Kolmogorov length scale of flow $(\sim 200 \mu \mathrm{m})$. The inter-droplet distance was evaluated based on the droplet number density $n$ calculated from PDA measurements, being $n^{-1 / 3}$ for a dilute spray [20]. In order to evaluate the Kolmogorov length scale, the integral time scale of the flow $T_{\text {turb }}$ was obtained by integrating the normalised autocorrelation coefficient of velocity measurement signal considering only droplets smaller than $10 \mu \mathrm{m}$. Thus, the integral length scale was evaluated as $L_{\text {turb }}=U_{b} T_{\text {turb }}$ by assuming that the Taylor hypothesis is valid.

The effect of incident laser on ignition was assessed by varying the laser's Q-switch delay, resulting in incident laser energies of 30, 40, 60, and $80 \mathrm{~mJ}$. The laser was fired at $2 \mathrm{~Hz}$ and at each laser shot, or ignition attempt, measurements of the incident laser energy $E_{i n}$ and the transmitted laser energy $E_{\text {trans }}$ were carried out using pyroelectric energy sensors and a beam splitter 
(Fig. 1, items 14-16). Thus, the instantaneous energy absorbed by the flow $E_{a b s}$ at each ignition attempt was evaluated as simply $E_{a b s}=E_{\text {in }}-E_{\text {trans }}$. One should notice that this definition of $E_{a b s}$ does not account for losses of energy along the beam path.

\subsubsection{Visualisation and velocity measurements}

Attention is given to the first jet diameter downstream of the nozzle: a region of $20 \times 20 \mathrm{~mm}$, where negligible entrainment and a uniform mean velocity profile are found (see Appendix). High-speed chemiluminescence of the $\mathrm{OH}^{*}$ radical and schlieren imaging were used to visualise the flame within this region. For the $\mathrm{OH}^{*}$ visualisation, a high-speed CMOS camera (Photron SA1.1) and a two-stage image intensifier (LaVision HS-IRO) were used, equipped with a Scheimpflug extension bellow, a 100-mm UV lens, and a $310 \mathrm{~nm}$ band-pass filter. The imaging of the flow was carried out at $12 \mathrm{kHz}$ with an exposure time of $80 \mu \mathrm{s}$. The recording of each ignition event was synchronised with the laser beam by using a photodiode, and then delayed by $100 \mu \mathrm{s}$ (except for Figs. 4 and 5), lasting for five milliseconds. The resulting size of the image was $20 \times 20 \mathrm{~mm}$, with a nominal resolution of $31.25 \mu \mathrm{m} /$ pixel. A simultaneous visualisation of the flow was done using the schlieren technique with a second Photron SA1.1 camera operating at the same settings. The light of a Xenon light source (Karl-Storz Xenon Nova 300) exiting a 1-mm optical fibre was collimated with a 200-mm plano-convex lens. The collimated light passed through the flow, and was refocused with a 500-mm plano-convex lens, passing through a $1.5-\mathrm{mm}$ pinhole, a long-pass 475-nm filter, and reaching the camera sensor. The imaging parameters (i.e. the frequency, exposure time, etc.) were kept the same as for the $\mathrm{OH}^{*}$ system.

A Dantec FiberFlow system was used for the velocity and droplet size measurements. The system was arranged in a 1-D LDA and PDA configuration, allowing for measurements of droplet diameters of up to approximately $80 \mu \mathrm{m}$ and axial velocity. The system consists of an Argon-Ion laser, a transmitting probe with a 500-mm focal length lens, a receiving probe with a 310-mm focal length lens and three photodetectors, which were positioned at an angle of $30^{\circ}$ off-axis from the forward scattering direction. Typical validation rates and spherical validation rates were above $95 \%$, with data rates of the order of $1 \mathrm{kHz}$. Approximately 20000 samples were obtained per position. The probes were mounted in a 3-dimension traverse, and measurements were taken across the jet for various axial distances, $z$, from the exit nozzle. 
The velocity of the gas phase was estimated by taking the velocity measurements of droplets smaller than $10 \mu \mathrm{m}\left(u_{z, g}=u_{z, d<10}\right)$ corresponding to a Stokes number of the order of 0.1. In this work, the over bar $\bar{y}$ and prime $y^{\prime}$ symbols denote the time-average and root mean square (rms) of $y$, respectively, while $\langle y\rangle$ represents a spatial-average of $y$ within the uniform part of the jet, that is, within $r<0.9 R$, approximately, where $R$ is the radius of the jet and $r$ is the radial coordinate.

\subsection{Experimental procedures}

Experiments were carried out to investigate how mixture, flow, and ignition source parameters affect the ignition process. Two sets of experiments were performed, and are detailed in Table 1 . The first set consists of experiments with a fixed flow bulk velocity, while the effect of varying the overall equivalence ratio was assessed. In the second set of experiments, the equivalence ratio was fixed at stoichiometry, and the flow bulk velocity was increased. In all experiments, two carrier flow preheating temperatures were investigated $\left(30\right.$ and $\left.50^{\circ} \mathrm{C}\right)$, and also four levels of laser energies $(30-80 \mathrm{~mJ})$. In total, 56 test conditions were investigated.

An important parameter in spray flames is the equivalence ratio of the gas phase, $\phi_{g}$, which is related to volatility of the liquid fuel and its prevaporisation upstream the region of interest. In this work, this parameter was explored in terms of the preheating temperature of the air flow, which controls the prevaporisation of the fuel inside the burner upstream the region of interest. As the experimental evaluation of $\phi_{g}$ is not trivial, a First-Law analysis of the problem was carried out (see Appendix). It was estimated that $\phi_{g}$ was 0.34 and 0.49 corresponding to the preheating temperature of air upstream of the atomiser of 30 and $50{ }^{\circ} \mathrm{C}$, respectively. Additionally, the outlet temperature of the gas for spray conditions could not be measured due to droplet interference on the probe. Still, temperatures of approximately 6 and $12{ }^{\circ} \mathrm{C}$ were estimated for low and high preheating conditions from the same analysis.

For each of the 56 test conditions, 120 ignition attempts were performed using the Nd-YAG laser. Statistics about the ignition process were obtained based on the energy measurements and $\mathrm{OH}^{*}$ visualisation. Two probabilities concerning the initial phases of the ignition process were defined: the probability of breakdown, $P_{b d}$, and the probability of ignition, $P_{i g n}$. In the present experiments, the initiation of the kernel depended solely on breakdown of the mixture by the laser, which was verified in the first frame of the $\mathrm{OH}^{*}$ 
Table 1: Summary of experimental conditions

\begin{tabular}{lr}
\hline Set \#1 of experiments & $6 \mathrm{~m} / \mathrm{s}$ \\
\hline Bulk flow velocity & $30,50^{\circ} \mathrm{C}$ \\
Preheat air temperature & $0.8,0.9,1,1.5,2$ \\
Equivalence ratio & $10,40,60,80 \mathrm{~mJ}$ \\
Laser ignition energy & 1 \\
\hline Set \#2 of experiments & $6,9,12 \mathrm{~m} / \mathrm{s}$ \\
\hline Equivalence ratio & $30,50^{\circ} \mathrm{C}$ \\
Bulk flow velocity & $30,40,60,80 \mathrm{~mJ}$ \\
Preheat air temperature & \\
Laser ignition energy & \\
\hline
\end{tabular}

sequence, while a successful ignition event was defined as the initiation of a kernel followed by its development into a self-sustained propagating flame. The growth of the flame was evaluated from the $\mathrm{OH}^{*}$ sequence: each image of the flame was binarised, and the area of the image $A_{f}$ corresponding to the line-of-sight visualisation of the flame was evaluated. Thus, an average radius of the flame $r_{f}(t)$ was simply obtained by assuming a spherical flame.

The systematic uncertainty [21] of the air mass flow rate was approximately $2 \%$ and $1 \%$ for the carrier and atomising air, respectively, $0.2 \%$ for the liquid mass flow rate, and $2{ }^{\circ} \mathrm{C}$ for temperature measurements (confidence interval of $95 \%$ ). For the energy measurements, the systematic uncertainty associated with the instruments was $3 \%$. The variation of the incident and absorbed energies are shown in Section 3.2. The combined uncertainty of $E_{a b s}$ (shown in Fig. 6) was evaluated using the Taylor series method for propagation of uncertainties described in [22].

Finally, calculations of the laminar planar and freely propagating gaseous premixed ethanol-air flame were carried out in the software Cosilab [23] using the Marinov chemical mechanism [24]. From these calculations, the laminar burning velocity for a stoichiometric condition $S_{L, s t}^{\circ}$ was evaluated as 0.42 and $0.46 \mathrm{~m} / \mathrm{s}$ for preheating temperatures of 30 and $50^{\circ} \mathrm{C}$, respectively. The unstretched laminar flame thickness was calculated from the temperature profile of the flame as $\left(T_{b}-T_{u}\right) / \max (\mathrm{d} T / \mathrm{d} x)[25]$.

\section{Results and discussion}

The results are presented as follows. First, a characterisation of the flow conditions is given, followed by the description of the phenomena observed in 
Table 2: Further details of experimental conditions, showing mean and rms of axial velocity, $\left\langle\bar{u}_{z, g}\right\rangle$ and $\left\langle u_{z, g}^{\prime}\right\rangle$, and $\left\langle d_{32}\right\rangle$ - all evaluated within the uniform region of the flow. Experiments \# $1-5$ and \#6-10 represent low and high preheating conditions, respectively, for various $\phi$. At the bottom of the table, experiments \#3-12 and \#8-14 represent increasing axial velocity for fixed low and high preheating temperatures, respectively.

\begin{tabular}{c|cccccc}
\hline$\#$ & $\begin{array}{c}\left\langle\bar{u}_{z, g}\right\rangle \\
(\mathrm{m} / \mathrm{s})\end{array}$ & $\begin{array}{c}\left\langle u_{z, g}^{\prime}\right\rangle \\
(\mathrm{m} / \mathrm{s})\end{array}$ & $\begin{array}{c}T_{\text {air }} \\
\left({ }^{\circ} \mathrm{C}\right)\end{array}$ & $\begin{array}{c}\phi \\
(-)\end{array}$ & $\begin{array}{c}\left\langle d_{32}\right\rangle \\
(\mu \mathrm{m})\end{array}$ & $\begin{array}{c}\frac{\left\langle u_{z, g}^{\prime}\right\rangle}{S_{L, s t}^{\circ}} \\
(-)\end{array}$ \\
\hline 1 & 6.2 & 0.4 & 30 & 0.8 & 25 & 0.9 \\
2 & 6.1 & 0.4 & 30 & 0.9 & 25 & 1.0 \\
3 & 6.1 & 0.4 & 30 & 1.0 & 25 & 1.0 \\
4 & 6.0 & 0.5 & 30 & 1.5 & 27 & 1.1 \\
5 & 6.0 & 0.5 & 30 & 2.0 & 28 & 1.2 \\
\hline 6 & 6.0 & 0.4 & 50 & 0.8 & 20 & 0.9 \\
7 & 6.1 & 0.4 & 50 & 0.9 & 20 & 0.9 \\
8 & 6.1 & 0.4 & 50 & 1.0 & 20 & 0.9 \\
9 & 6.2 & 0.5 & 50 & 1.5 & 22 & 1.0 \\
10 & 6.4 & 0.5 & 50 & 2.0 & 24 & 1.1 \\
\hline \hline 3 & 6.1 & 0.4 & 30 & 1.0 & 25 & 1.0 \\
11 & 10.5 & 0.5 & 30 & 1.0 & 27 & 1.2 \\
12 & 13.1 & 0.6 & 30 & 1.0 & 27 & 1.3 \\
\hline 8 & 6.1 & 0.4 & 50 & 1.0 & 20 & 0.9 \\
13 & 10.3 & 0.6 & 50 & 1.0 & 24 & 1.3 \\
14 & 12.9 & 0.6 & 50 & 1.0 & 26 & 1.3 \\
\hline
\end{tabular}

the experiments, which illustrate the randomness of the process and define its timescales. A statistical analysis of kernel sizes and timescales of ignition is given next, showing the influence of laser ignition energy and of equivalence ratio on these parameters. Finally, measurements of probability of breakdown and ignition are presented.

\subsection{Flow measurements}

The main results of the characterisation of the flow field are presented in this section, and a detailed discussion on the uniformity of the flow can be found in the Appendix. Figure 2 shows the (a) turbulence intensity and the (b) Sauter mean diameter $\left(d_{32}\right)$ of the droplets for the first set of experiments. Turbulence intensities generally increased with $\phi$ (Fig. 2a), from $6 \%$ to $8 \%$. Inside the burner, turbulence was generated through the high shear between the spray cone and the carrier flow, and higher air flow rates in the air- 


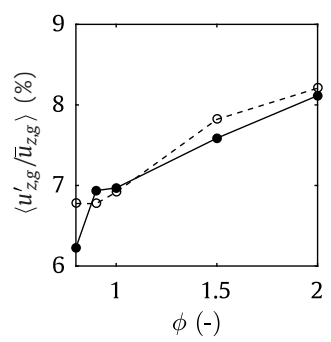

(a)

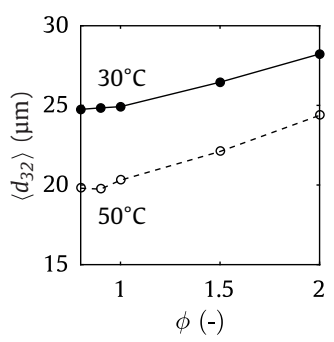

(b)

Figure 2: The (a) turbulence levels and (b) Sauter mean diameter for 30 and $50^{\circ} \mathrm{C}$ preheat temperature, with varying equivalence ratio $-U_{b}=6 \mathrm{~m} / \mathrm{s}, r=0$.

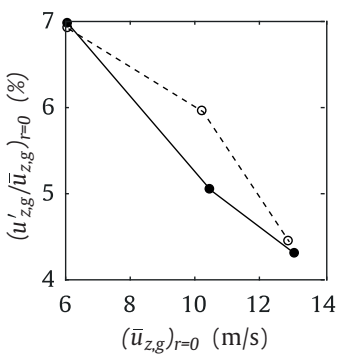

(a)

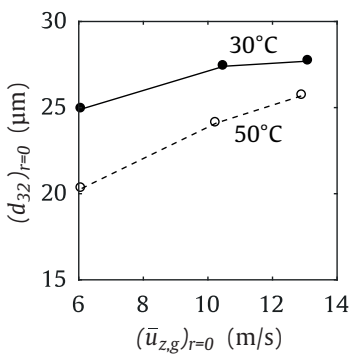

(b)

Figure 3: The (a) turbulence levels and (b) Sauter mean diameter evaluated at the centre of the jet $(r=0)$, for 30 and $50{ }^{\circ} \mathrm{C}$ preheat temperature, with varying bulk flow velocity $-\phi=1$.

assist atomiser were needed to atomise high liquid flow rates. Efforts were made to keep the axial velocity constant, within $\pm 5 \%$ of $6.1 \mathrm{~m} / \mathrm{s}$, accounting for the influence of evaporative cooling occurring inside the burner, changes in the atomising air, and different preheating temperatures. The effect of increasing the preheating temperature consistently decreased $d_{32}$, although some variation was observed due to changes in atomisation with equivalence ratio. A summary of the experimental conditions is shown in Table 2.

Both $u_{z, g}^{\prime} / \bar{u}_{z, g}$ and $d_{32}$ are also shown for the experiments with varying bulk flow velocity (Fig. 3). Turbulent velocity fluctuations increased with axial velocity (Table 2), allowing for experiments where the effect of increasing turbulence and reducing prevaporisation of the flow on ignition was evaluated. The investigated range of $\left\langle u_{z, g}^{\prime}\right\rangle / S_{L, s t}^{\circ}$ was limited (0.9-1.3, Table 2), corresponding to a regime of weakly corrugated premixed flame. This suggests that the effects of flow parameters on ignition and breakdown prob- 


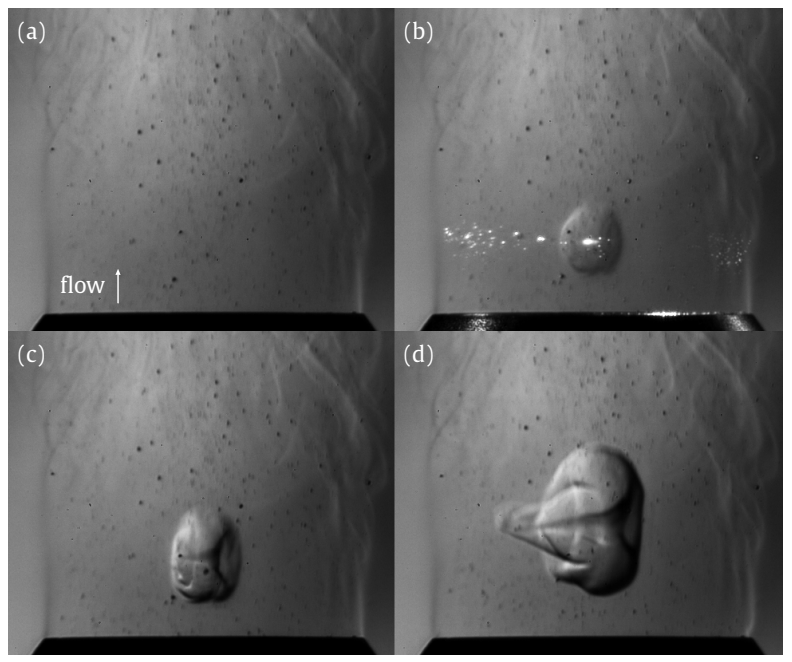

Figure 4: Schlieren image sequence illustrating the ignition process: (a) droplet-laden flow, (b) laser spark, (c) flame kernel, and (d) growth and convection of the flame downstream the jet $\left.-U_{b}=6 \mathrm{~m} / \mathrm{s}, \phi=1.5, T_{i n}=30^{\circ} \mathrm{C}, E_{\text {in }}=60 \mathrm{~mJ}\right)$.

ability are more likely to be traceable to overall equivalence ratio, SMD, and small-scale wrinkling associated with the spray, rather than high turbulent strain.

\subsection{Kernel formation and ignition}

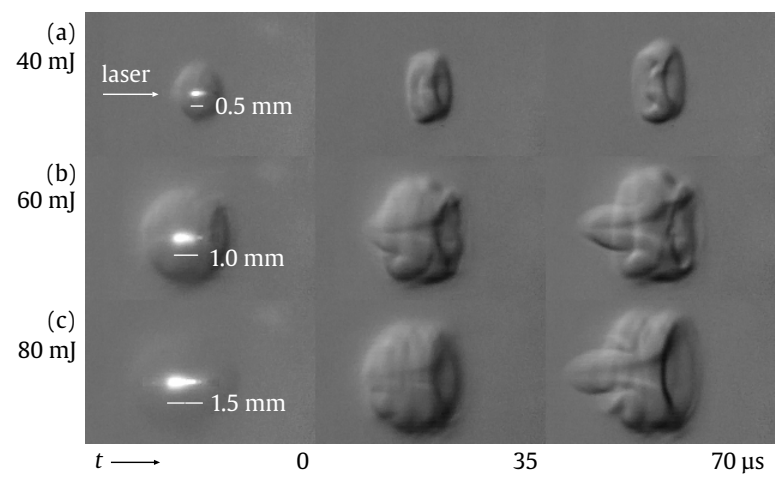

Figure 5: Schlieren visualisation of the breakdown and following $70 \mu$ s after a spark event in quiescent air for laser energy of (a) $40 \mathrm{~mJ}$, (b) $60 \mathrm{~mJ}$, (c) $80 \mathrm{~mJ}$.

The first instants following the spark are shown in the schlieren imaging of Fig. 4. The laser beam, focused at the centre of the flow and $5 \mathrm{~mm}$ 
downstream of the nozzle, caused breakdown the mixture (b) and generated a flame kernel that grew (c) and convected downstream of the jet (d). Due to the long exposure $(80 \mu \mathrm{s})$ of the schlieren visualisation, frame (b) shows simultaneously the scattering of the beam due to the droplets, the bremsstrahlung radiation emitted by the plasma at the focusing point, and the initial kernel. Increasing the incident energy of the laser lengthened the plasma from 0.5 to $1.5 \mathrm{~mm}$ as seen in Fig. 5 for a spark in quiescent air, which then led to larger initial kernels. Such kernels were large compared to the interdroplet distance, thus it is expected that fuel vapour fluctuations in the interdroplet space did not affect the generation of the kernel.

Part of the randomness of the laser ignition process of spray flows is illustrated in Fig. 6, which shows in (a) the shot-to-shot variation of the incident laser energy $E_{i n}$ and the absorbed energy by the flow $E_{a b s}$. The result of the spark event is depicted by different markers representing three possible outcomes: ignition, kernel formation and subsequent quenching, and no kernel formation (i.e. no breakdown). No evident relation between the fluctuations of $E_{i n}$ and $E_{a b s}$ was observed (i.e. not constant $E_{a b s} / E_{i n}$, nor between $E_{a b s}$ and the outcome of the spark event. Conditional averages of $E_{a b s}$ based on the spark outcome are depicted as horizontal lines. The average value of $E_{a b s}$ concerning no-breakdown cases offers a reasonable estimation of the energy lost due to absorption and Mie scattering along the beam path. Hence, for this condition, it can be estimated that only approximately half of $E_{a b s}$ contributed to breakdown of the mixture. No significant difference between the average $E_{a b s}$ corresponding to ignition and to quenched events was observed in this condition.

Further, the resulting kernels of a successful breakdown event presented variation in density of the $\mathrm{OH}^{*}$ radical and size, which was found to strongly affect the outcome of an ignition event, as discussed further in Sec. 3.3. These variations are shown in Fig. 6b, with flame kernels imaged $100 \mu$ s after the spark, for specific ignition attempts chosen from Fig. 6a. These events were grouped by similarity in the $E_{a b s}$ plot: events 17 and 33 presented a peak value of $E_{a b s}$ and resulted in successful ignition, while 16 and 60 resulted in the quenching of the flame even though $E_{a b s}$ was also high in both events. Although these events presented kernels of similar size, events 17 and 33 were characterised by a much stronger $\mathrm{OH}^{*}$ signal, indicating higher reaction rates in the flame kernel. In contrast, events 22 and 80 show small kernels with low $\mathrm{OH}^{*}$ intensity that still resulted in ignition, although the respective $E_{a b s}$ values were low and similar to events where breakdown was not observed. 


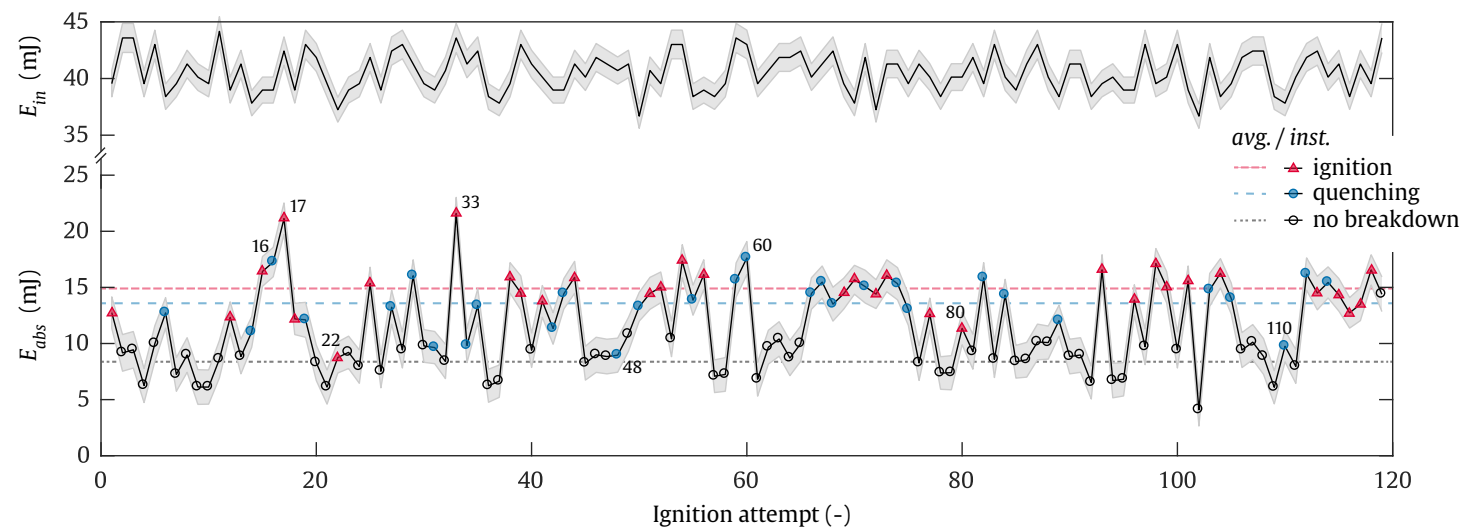

(a)
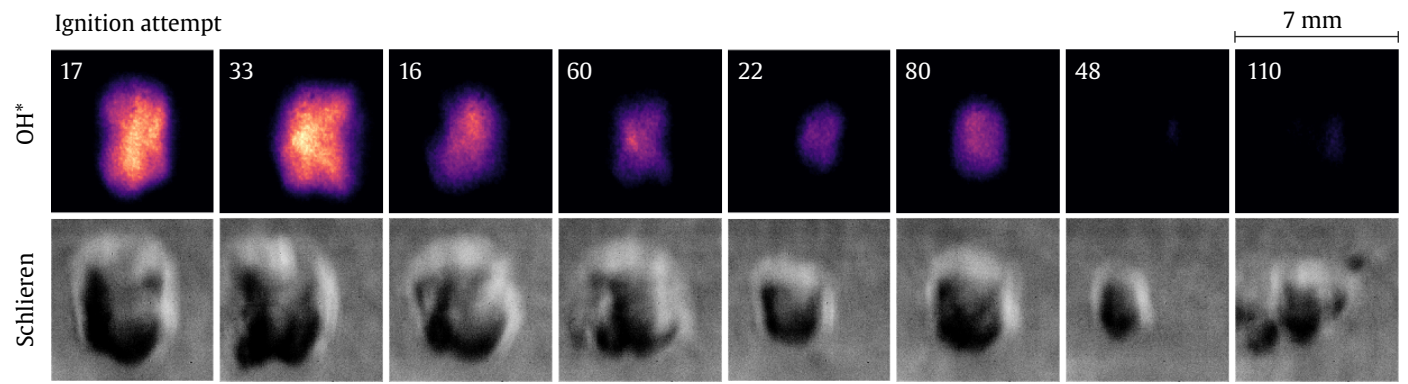

(b)

Figure 6: Ignition attempts in a flow $-U_{b}=12 \mathrm{~m} / \mathrm{s}, \phi=1, T_{i n}=30^{\circ} \mathrm{C}$. (a) The incident laser energy and absorbed energy, and their respective systematic and expanded uncertainty values (shown in light grey). Conditional averages based on ignition, quenching, and no breakdown are shown in horizontal lines. (b) $\mathrm{OH}^{*}$ and schlieren visualisation at $t=0.1 \mathrm{~ms}$ for selected ignition attempts based on the value of $E_{a b s}$ and the outcome of the event as defined in (a).

Finally, 48 and 110 show small kernels that quenched, but were identified through the first frame of the $\mathrm{OH}^{*}$ sequence and through schlieren.

Following the formation of a kernel, its development into a self-sustained flame is illustrated in Figure 7. The average radius of the flame $r_{f}$ as a function of time after the spark event $t$ is shown in Fig. 7a, with each curve representing one distinct ignition attempt. Events where the kernel developed into a self-sustained flame are marked in red, representing ignition, while events where quenching occurred are marked in blue. This notation is 
used throughout this work. In this figure, the effect of increasing the incident energy on the total number of kernels can be clearly observed, while a significant increase of the number of kernels that ignite only occurred for $80 \mathrm{~mJ}$. Additionally, other effects of laser energy on the flame growth can be observed: successful ignition events with higher energy, for example, presented a steeper slope of $r_{f}$ until $t=0.2 \mathrm{~ms}$, indicating the persistence of the flame overdrive effect due to the laser spark [19]. This effect seemed to strongly affect the initial size of the kernels, due to a combination of increasing the plasma size and, consequently, the absorbed energy as the incident energy increases, with the flame overdrive that accelerates the growth of the kernel immediately after the spark.

The phenomena shown in Fig. 7 (a) are summarised in (b). The flame kernel can ignite (b, i) or quench by distinct modes which are characterised by their timescales: (ii) long and (iii) short mode of ignition failure, as defined by Mastorakos [5]. Examples of events where ignition and long and short mode failure were observed are shown by means of $\mathrm{OH}^{*}$ and schlieren visualisation sequences in Fig. 7c. The successful ignition attempt was seen both in the $\mathrm{OH}^{*}$ and in the schlieren sequences as a kernel that grew radially and convected downstream (upward). In the failed attempts, the $\mathrm{OH}^{*}$ signal decreased to zero, although in some events the schlieren sequence showed some growth of the spherical region due to the heat transfer between the non-reacting hot gas kernel and the surrounding flow. Additionally, the uneven $\mathrm{OH}^{*}$ signal distributed within the kernel with small regions of high concentration, suggests that for this case of low prevaporisation the flame was characterised by individual droplet burning as well as flame propagation through the interdroplet spacing. For a similar condition, DNS [26] showed that successful ignition was due to the proximity of the droplets at the spark region, which then generated a flame front that vaporised the fuel ahead of it creating a flammable interdroplet spacing and, therefore, characterised by small areas of high heat release in the vicinity of droplets and large areas of low heat release in the interdroplet spacing.

The majority of short-mode failure events were characterised by an initially low $\mathrm{OH}^{*}$ signal in the kernel (Fig. 7c, iii), indicating low temperatures and heat release rates following the deposition of energy by the spark. This mode of ignition failure was observed in $[8,17]$ and attributed to a locally lean mixture and consequently small size of the kernel, which did not allow for thermal runaway to occur despite the initial temperatures above the adiabatic flame temperature. In this case, for ignition to occur, a higher 
spark energy would be required [27]. Long-mode failure events commonly presented higher values of $\mathrm{OH}^{*}$ immediately after the spark in the present experiments, indicating that combustion took place. In such cases, failure to ignite has been attributed to the presence of liquid either within the core or close to the flame front [17], preventing the flame from propagating due to intense local evaporative cooling.

A precise experimental evaluation of the quenching timescales of short and long mode failure events depends on the definition of the timescale of the spark itself. Although the duration of the laser pulse was between 4-6 ns, the resulting effects of the spark on the flame were longer than the timescale of the plasma, the latter being typically $1 \mu \mathrm{s}$ [28]. Thus, a critical time $t_{\text {crit }}$ was defined as the time at which the effects of the spark overdriving the chemical reactions in the flame have decayed. This timescale has been measured previously in gaseous mixtures based on radical emissions [29, 30], and defined as a net increase of chain-branching reactions in the flame kernel following the decrease in radical emissions occurring during the plasma-cooling dominated phase. The parameter $t_{\text {crit }}$ was used to distinguish between the two modes of failure: events that were shorter or longer than $t_{c r i t}$ were defined as being of short-mode and long-mode failure, respectively. It should be noted that the present evaluation of $t_{\text {crit }}$ does not account for additional gas dynamic effects [19] that also enhance the propagation of the flame.

In the present work, the chemiluminescence of the $\mathrm{OH}^{*}$ radical was used as an indicator of the reaction rate of the flame [31], so that $t_{\text {crit }}$ could be evaluated. Figure 8 a shows the normalised $\mathrm{OH}^{*}$ density, $\Gamma^{\prime}$, for a specific test condition and multiple ignition events. This parameter was evaluated as,

$$
\begin{gathered}
\Gamma^{\prime}(t)=\frac{\Gamma}{\Gamma(100 \mu s)} \\
\Gamma(t)=\frac{1}{A_{f}} \int_{A_{f}} I_{O H^{*}} \mathrm{~d} A
\end{gathered}
$$

where $I_{O H^{*}}$ represents the intensity of the $\mathrm{OH}^{*}$ radical as imaged by the camera sensor. An average curve of $\Gamma^{\prime}$ was evaluated, and $t_{\text {crit }}$ was calculated as the time at which $\Gamma^{\prime}$ has reached $1 \%$ of its asymptote value, meaning the spark effects were no longer acting on the flame at this point.

Figure $8 \mathrm{~b}$ shows an effect of the spark on the flame up to until $0.7 \mathrm{~ms}$ after the spark, as seen in the case of the leanest equivalence ratio and highest 


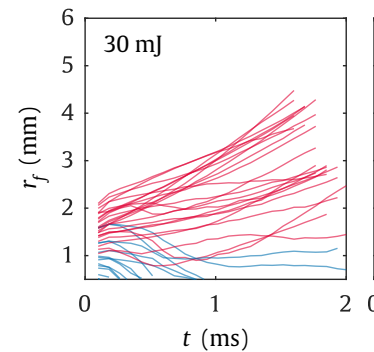

(c)

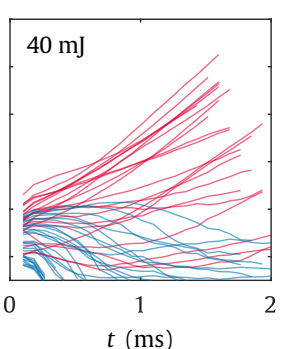

20

(a)

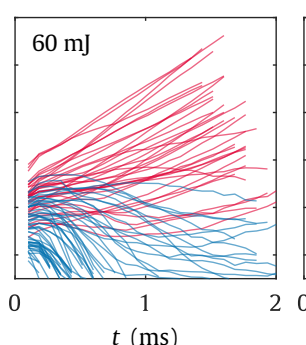

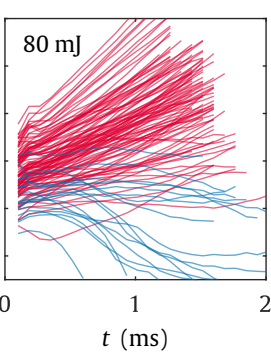
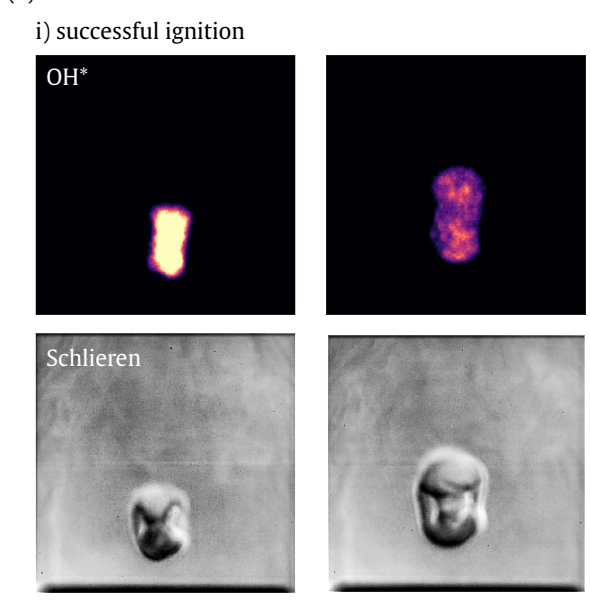

ii) long mode of ignition failure
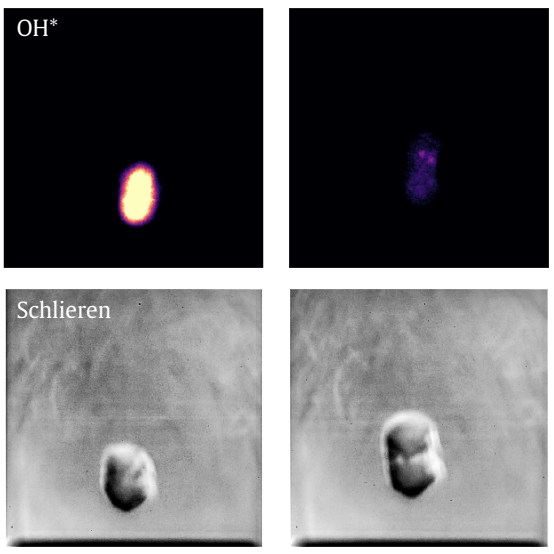

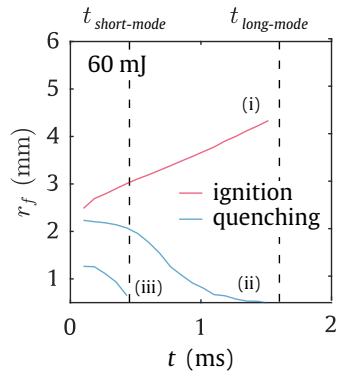

(b)
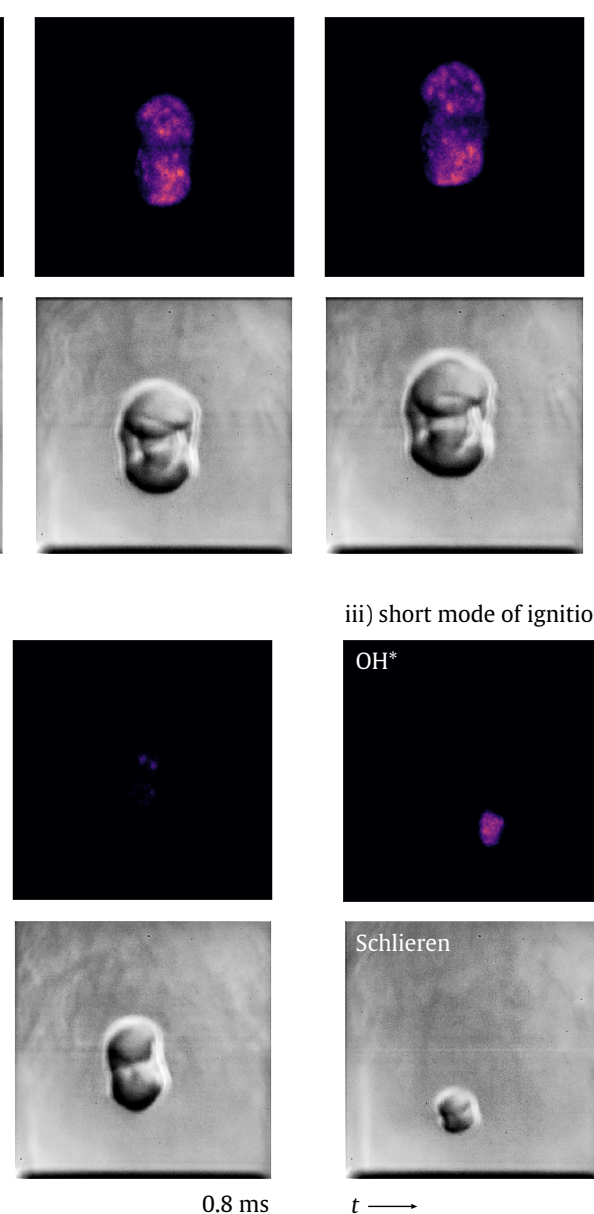
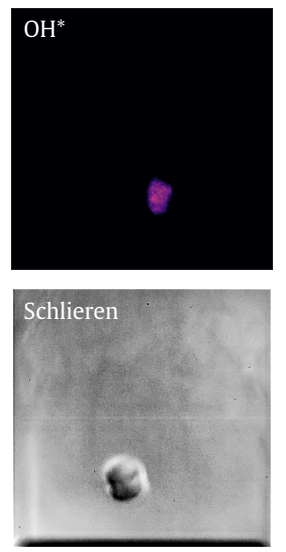

$t \longrightarrow$
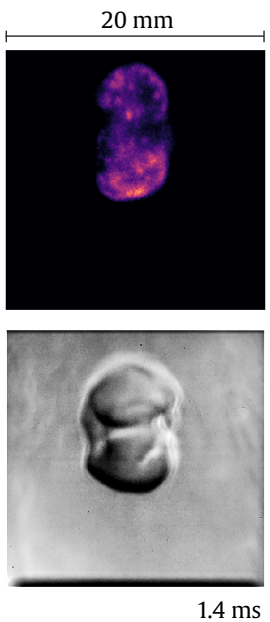

iii) short mode of ignition failure
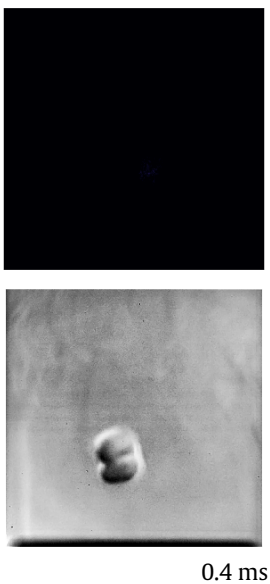

Figure 7: Example of (a) a series of successful (red) and failed (blue) ignition attempts based on the growth of the kernel radius $r_{f}$ for various levels of laser energy $-U_{b}=6 \mathrm{~m} / \mathrm{s}$, $\phi=1, T_{\text {in }}=30^{\circ} \mathrm{C}$. Three events given in (b) comprising one successful ignition event (i) and two failed ignition events (ii,iii) are shown in (c) by means of schlieren and $\mathrm{OH}^{*}$ visualisation. 


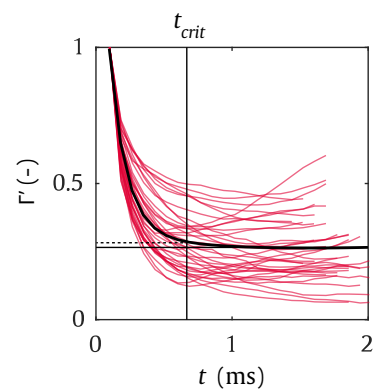

(a)

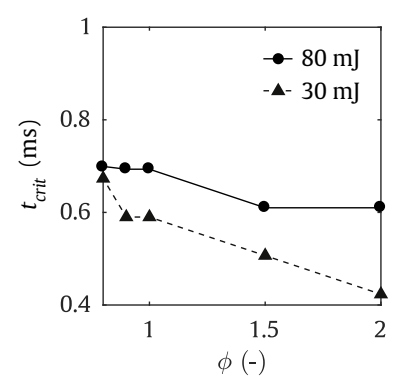

(b)

Figure 8: Calculation of $t_{\text {crit }}$ based on the normalised density of the $\mathrm{OH}^{*}$ signal over time $\left(U_{b}=6 \mathrm{~m} / \mathrm{s}, \phi=1, T_{i n}=30^{\circ} \mathrm{C}, E_{\text {in }}=60 \mathrm{~mJ}\right)$, and (b) $t_{\text {crit }}$ in terms of equivalence ratio for the lowest and highest ignition energy cases $\left(T_{i n}=50^{\circ} \mathrm{C}\right)$.

laser energy. This value was lower than the chemical timescale of the flame calculated for the present experiment, $t_{f} \cong 1 \mathrm{~ms}$, evaluated as $\delta / S_{L}^{\circ}$. The overdrive effect was also assessed in DNS [8] based on the temperature of the kernel, and showed that within $0.6 t_{f}$ from the maximum temperature of the kernel normal adiabatic flame temperatures were reached for cases of $\phi$ varying from 0.5 to 1.5 . In the present experiments, the overdrive effect was verified to be of very similar duration, ranging from 0.4 to $0.7 t_{f}$. Nevertheless, values of $t_{\text {crit }}$ were found to be slightly higher for cases with high energy $(80 \mathrm{~mJ})$, decreasing with overall equivalence ratio especially for low energy cases. Additionally, $t_{\text {crit }}$ in ethanol sprays were longer than those reported by [30] for an air-methane mixture, which were approximately $200 \mu$ s for $\phi$ between 0.7 and 1 [30].

\subsection{Statistics of timescale and kernel size}

Distributions of initial kernel size are shown in Fig. 9 for conditions of low preheat temperature and bulk velocity of $6 \mathrm{~m} / \mathrm{s}$. The density functions of kernel size were estimated from the data histogram and plotted separately according to ignition or quenching depicted in shades of red and blue, respectively, and according to $\phi$. The density curves were obtained through the Parzen-Rosenblatt window method [32, 33], which allows for the representation of the histogram shape, as this method does not assume an underlying distribution of the dataset. Additionally, the integration of the given density curves over the horizontal-axis parameter simply leads to the total number of events observed, as opposed to unity as in the case of a probability density 

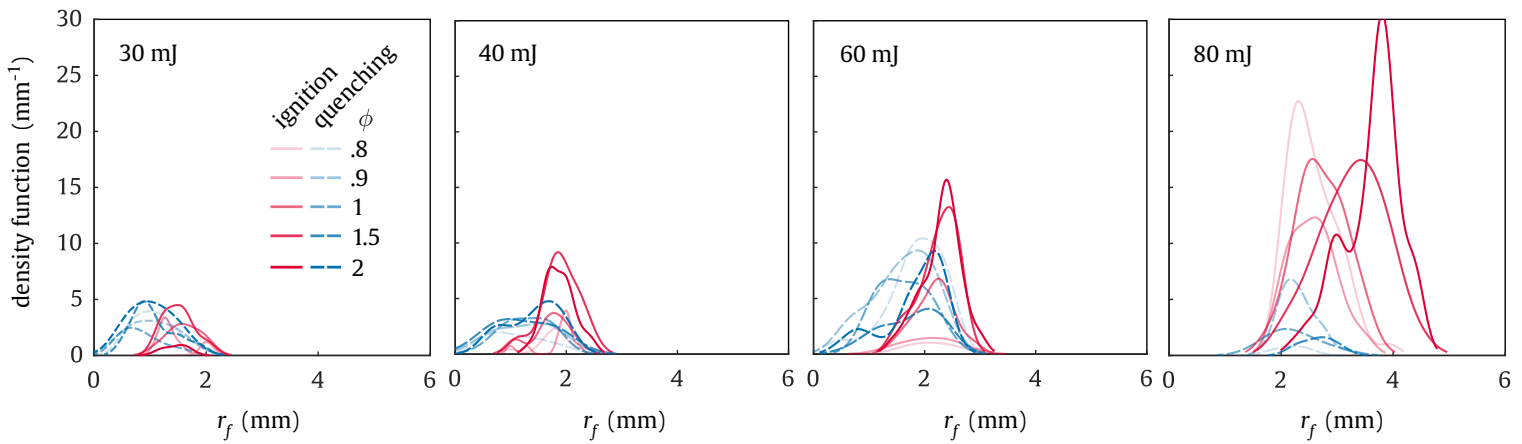

Figure 9: Influence of ignition energy and equivalence ratio on initial kernel size distribution and ignition $-U_{b}=6 \mathrm{~m} / \mathrm{s}, T_{i n}=30^{\circ} \mathrm{C}$.
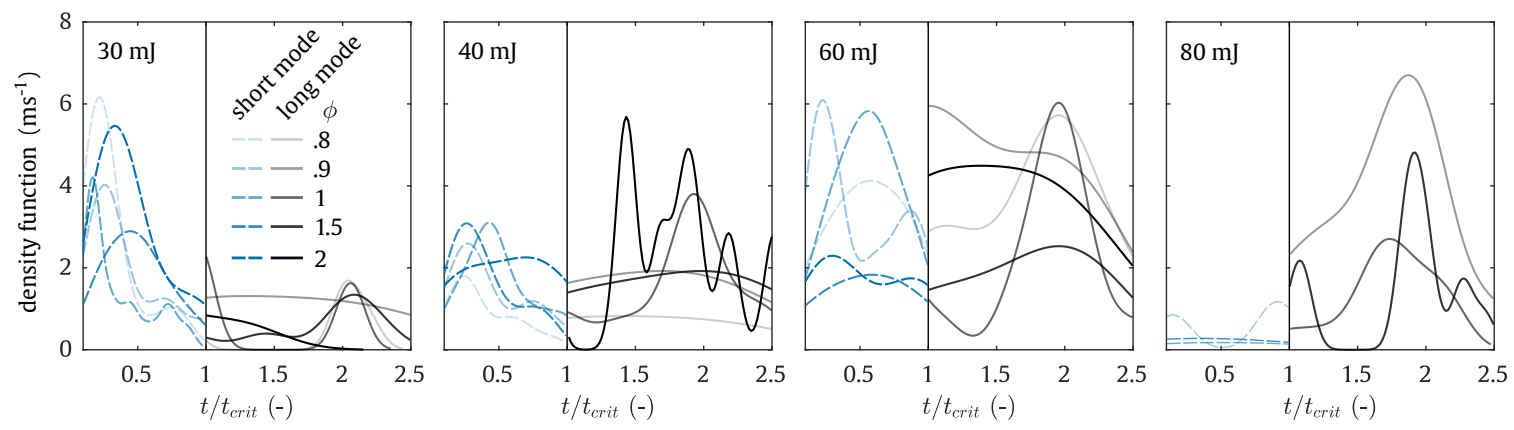

Figure 10: Distributions of short and long mode of ignition failure timescales for various energy levels and equivalence ratios $-U_{b}=6 \mathrm{~m} / \mathrm{s}, T_{i n}=30^{\circ} \mathrm{C}$.

function. A strong dependency of the initial kernel size with $E_{\text {in }}$ was observed, with kernel size increasing in almost three times as energy increased from 30 to $80 \mathrm{~mJ}$. Additionally, a minimum kernel size for ignition was observed for $E_{\text {in }}=30$ to $60 \mathrm{~mJ}$, being approximately $1 \mathrm{~mm}$. This value closely agrees with the theoretical minimum kernel size for a spherical kernel in a gaseous mixture [34], defined as two times the flame thickness $(\delta \cong 0.5 \mathrm{~mm}$, for an equivalent gaseous stoichiometric mixture). Such limit was not observed for $80 \mathrm{~mJ}$, as the sparks led to kernels larger than $1.5-2 \mathrm{~mm}$. For this level of incident energy, the long-mode of ignition failure was observed, and will be discussed next. In general, the effect of equivalence ratio on the initial kernel radius seemed to affect mostly the total number of events, but not the range of kernel sizes. However, for $80 \mathrm{~mJ}$, there is a clear dependency of $r_{f}$, 


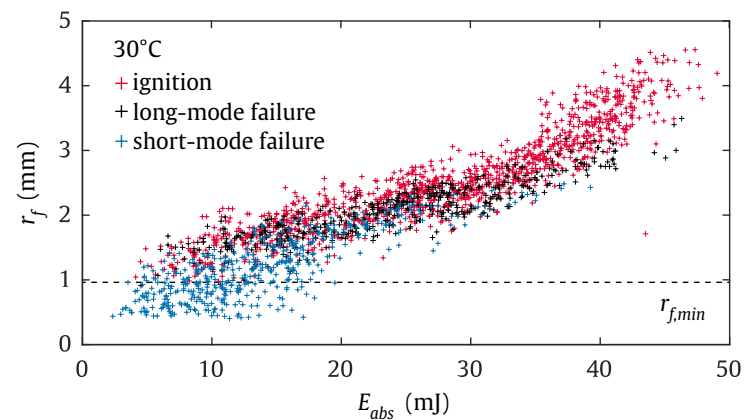

(a)

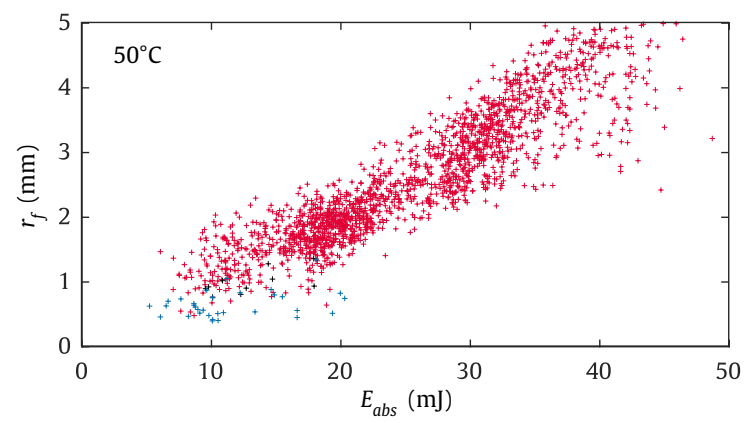

(b)

Figure 11: Initial kernel size in terms of the absorbed energy for preheat temperature of (a) $30^{\circ} \mathrm{C}$ and (b) $50{ }^{\circ} \mathrm{C}-U_{b}=6 \mathrm{~m} / \mathrm{s}$

which increased with $\phi$.

Further, Fig. 10 shows distribution functions of ignition failure timescales normalised in terms of $t_{c r i t}$. In each plot, distinct density functions are presented for short and long mode, depicted in shades of blue and grey, respectively, according to $\phi$. Results show substantial change from short to long-mode failure as $E_{\text {in }}$ increased, with short-mode quenching being fully suppressed at $80 \mathrm{~mJ}$. Additionally, in experiments with preheating of $50^{\circ} \mathrm{C}$ both short and long mode failures were scarce, as failure to ignite the flow was almost exclusively due to failure to break down the mixture. This will be discussed in Sec. 3.4.

The relation between the absorbed laser energy $E_{a b s}$ and the initial radius of the flame is given by Figure 11, with red markers representing ignition events, and blue and black markers representing short and long-mode failure events, respectively. For the low preheating condition (Fig. 9a), the presence of the minimum kernel radius for values below $E_{a b s} 20 \mathrm{~mJ}$ can be clearly 
noticed. Nevertheless, the presence of short-mode failure events for values of $r_{f}$ above the minimum size and up to more than twice that value indicate that low reaction rates (Fig. 7) and other mechanisms other than the initial kernel size may also have resulted in short-mode failure in the present experiment.

Figure 11 presents a positive correlation between the initial radius of the flame and the outcome of the ignition event, showing that the number of ignition events increases with the initial kernel radius. Additionally, a suppression of all quenching modes was observed once the preheating temperature was increased to $50^{\circ} \mathrm{C}$, that is, as the equivalence ratio of the gas phase increased to approximately 0.5 , being closer to the lower flammability limit of 0.66 . The effects of the kernel radius and prevaporisation indicate that the presence of a flammable mixture in the spark location is determining to ignition, as shown in DNS [35]. The probability of finding a flammable mixture in the spark not only increases with prevaporisation, but should also increase with the spark size as a large spark will experience less fuel fluctuations. This affects polydisperse sprays, in which a significant fraction of the injected fuel is carried by large droplets which are scarce in the flow, as shown in Fig. 17 of the Appendix.

\subsection{Ignition probabilities}

The probabilities of kernel formation and ignition in terms of equivalence ratio are shown in Fig. 12. For ignition energies between 30 and $60 \mathrm{~mJ}$, an increase in equivalence ratio generally resulted in an increase of $P_{b d}$. Such behaviour was expected, as it is known that the presence of droplets along the beam path can act as micro lenses facilitating breakdown around the focusing point of the beam [36]. Therefore, increasing the total amount of liquid, which effectively led to an increase of the droplet number density, enhanced the probability of breakdown, or $P_{b d}$. However, this effect was balanced by energy losses along the beam path between the focusing lens and the ignition location, being detrimental to $P_{b d}$. The lost energy as the laser beam crosses the flow was estimated from the mean value of $E_{a b s}$ conditional to events where no breakdown was observed, shown in Fig. 13. The results indicate the competing effects described previously, showing that losses increased with equivalence ratio and also with preheating temperature - the latter being likely associated with the suppression of the droplet-enhancement effect of breakdown, which increased the average breakdown threshold.

An estimate of the actual energy deposited at the focusing point, $E_{a b s}^{*}$, was evaluated by taking the mean value of $E_{a b s}$ conditional to events where no 


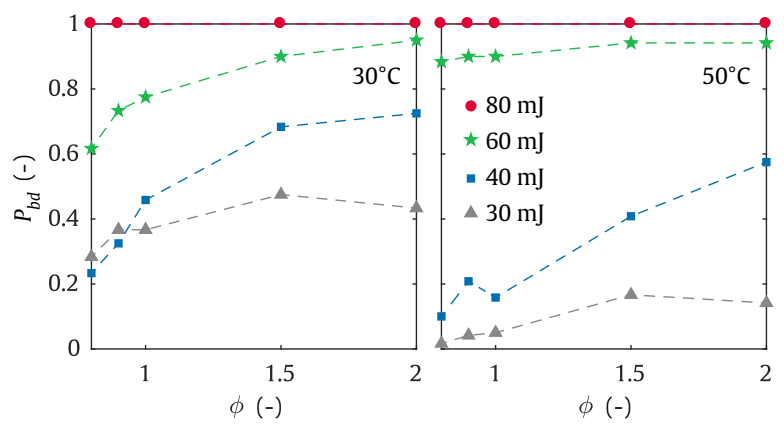

(a)

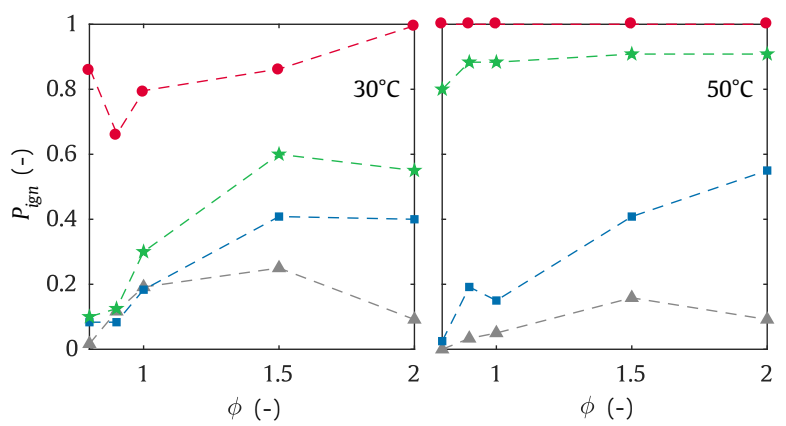

(b)

Figure 12: Probabilities of (a) kernel formation and (b) ignition for $U_{b}=6 \mathrm{~m} / \mathrm{s}$ and $T_{\text {in }}=30$ and $50^{\circ} \mathrm{C}$.

breakdown was observed and subtracting it from the mean value of the same parameter conditional to events corresponding to breakdown. These values are reported in Fig. 14. Nevertheless, it should be noted that large experimental uncertainties associated with the evaluation of $E_{a b s}^{*}$ are expected as a result of the combined uncertainty deriving from the energy measurements $(1-4 \mathrm{~mJ})$, as well as due to the random variations of the measured and derived parameters inherent of such experiments in sprays, as illustrated previously in Fig. 6. Values of $E_{a b s}^{*}$ varied between 1 and $20 \mathrm{~mJ}$ in the experiments, while the threshold energy for breakdown of air and fully-prevaporised ethanol-air mixtures was measured $\left(P_{b d}=0.5\right)$ as approximately $8.5 \mathrm{~mJ}$. This seems to explain the effect of prevaporisation on $P_{b d}$ (Fig. 12), as increasing prevaporisation resulted in an overall higher $P_{b d}$ for high $E_{i n}$, but lower $P_{b d}$ for low $E_{\text {in }}$. For $E_{\text {in }}$ resulting in a deposited energy in the focusing point below the breakdown energy for the gaseous mixture, the creation of a flame kernel relied on the presence of droplets to promote breakdown. Therefore, $P_{b d}$ 

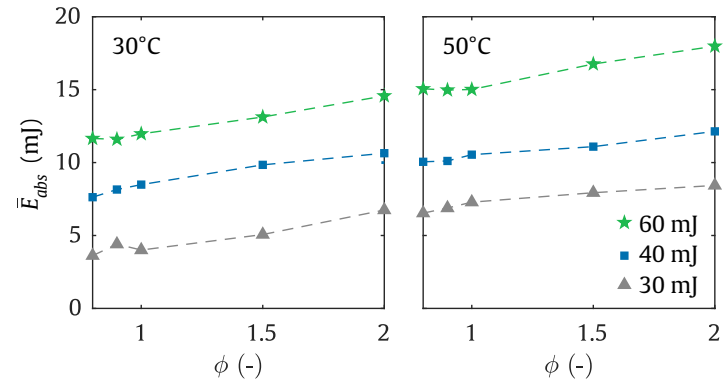

Figure 13: Mean value of $E_{a b s}$ conditional to no-breakdown events, representing energy losses along the beam $-T_{i n}=30$ and $50^{\circ} \mathrm{C}, U_{b}=6 \mathrm{~m} / \mathrm{s}$

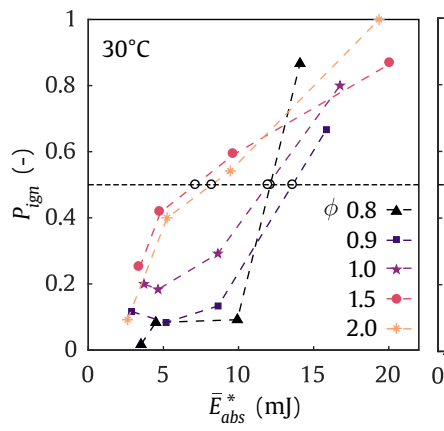

(a)

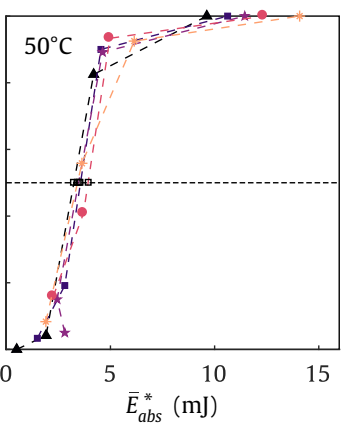

(b)

Figure 14: Probability of ignition in terms of the energy deposited at the spark location, $E_{a b s}^{*}$, for various equivalence ratios and (a) $T_{i n}=30^{\circ} \mathrm{C}$ and (b) $50{ }^{\circ} \mathrm{C}\left(U_{b}=6 \mathrm{~m} / \mathrm{s}\right)$. Minimum ignition energy is shown as $E_{a b s}^{*}\left(P_{i g n}=0.5\right)$ for each condition.

decreased as the smallest droplets of the flow fully vaporised with preheating, resulting in a significantly lower droplet number density. In contrast, a high $E_{i n}$ was not affected by prevaporisation, hence a unity $P_{b d}$ was verified for $80 \mathrm{~mJ}$ and all $\phi$. It should be noted that consistently-defined MIE for ethanol vapour at the conditions studied $(p, T)$ were not available, and so a direct comparison with gaseous-only systems is not possible at present. Still, the trends with preheating provide useful insight.

Figure 12 also shows that lean conditions with low prevaporisation required the highest energy to ignite, which agrees with DNS [27]. Increasing $E_{\text {in }}$ from 30 to $60 \mathrm{~mJ}$ gradually facilitated the formation of kernels, but their subsequent quenching was still verified, hence resulting in virtually no changes to $P_{i g n}$. Furthermore, only a significant increase of the ignition energy to $80 \mathrm{~mJ}$ was effective in suppressing quenching. Increasing the amount 


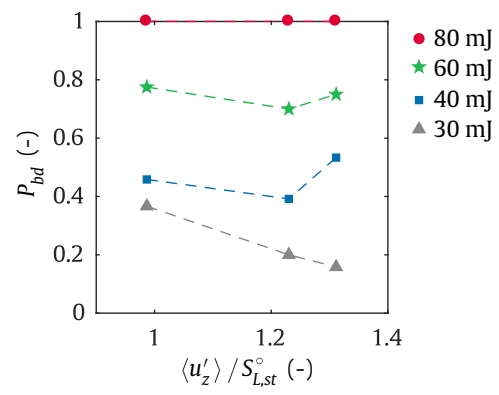

Figure 15: Probability of breakdown and ratio of probability of ignition in terms of probability of breakdown $-U_{b}=6,9$, and $12 \mathrm{~m} / \mathrm{s} ; T_{i n}=30^{\circ} \mathrm{C}$.

of prevaporised fuel in lean conditions decreased this threshold to $60 \mathrm{~mJ}$, as more of energy due to combustion was available to overcome evaporation and less fuel was present as liquid. Further, the minimum ignition energy evaluated as $E_{a b s}^{*}\left(P_{i g n}=0.5\right)$ is shown in Fig. 14. A consistent value of approximately $3.5 \mathrm{~mJ}$ was obtained for high prevaporisation and lower $d_{32}$, while higher values between 4 and $14 \mathrm{~mJ}$ were observed for conditions of low prevaporisation. For high prevaporisation, little effect of $\phi$ on $P_{i g n}$ was noticed, as the mixture in the interdroplet region was close to flammable and no failure of the kernel occurred once a kernel was formed. Further, for both conditions (a) and (b), the obtained MIE is higher than the theoretical limit of $1.5 \mathrm{~mJ}$ evaluated according to [34], which has been attributed not only to the effects of spray and turbulence, but also due the added energy released in the form of a shock wave that propagates from the spark [37].

The effect of turbulence on $P_{b d}$ is shown in Fig. 15. Significant changes were mostly noticed for a low ignition energy $(30 \mathrm{~mJ})$, where the effect of turbulence seemed strongly detrimental to $P_{b d}$. As $E_{i n}$ increased, resulting in larger kernels, this effect became less pronounced and completely vanished once energy reached $80 \mathrm{~mJ}$. It should be noted that other than the effect of turbulence, some decrease of $\phi_{g}$ is expected as $\left\langle u_{z, g}^{\prime}\right\rangle / S_{L, s t}^{\circ}$ increases due to changes of residence time of the droplets inside the burner. Further work is needed with higher turbulence and controlled $\phi_{g}$ to allow for a more detailed analysis.

\section{Conclusions}

The problem of a spherically expanding flame was investigated in a turbulent jet consisting of a uniform distribution of ethanol droplets in a fuel 
vapour-air mixture. The flow exhibited a top-hat velocity profile and a uniform polydisperse droplet distribution in the region where the flame was visualised. The ethanol spray followed a modified Rosin-Rammler distribution with Sauter mean diameter values of approximately $25 \mu \mathrm{m}$, which decreased by $20 \%$ with preheating of the air flow. Characteristic values of $\left\langle u_{z, g}^{\prime}\right\rangle / S_{L, s t}^{\circ}$ were between 0.9 and 1.3 , corresponding to the regime of weakly corrugated premixed flame.

The probability of breakdown $P_{b d}$ was evaluated from the first frame of the $\mathrm{OH}^{*}$ image sequence and depended on the laser beam promoting breakdown of the mixture at the focusing point. In the experiments, $P_{b d}$ was mainly controlled by two competing effects that increased with equivalence ratio: the enhancement of breakdown due to the presence of droplets in the focusing point of the laser, and energy losses due to Mie scattering and absorption occurring along the beam path. Overall, $P_{b d}$ was improved by increasing the equivalence ratio of the mixture, while the effect of prevaporising the fuel was overall detrimental to $P_{b d}$ as it resulted in a decrease of the droplet number density. For high incident laser energies, breakdown was independent of the presence of droplets in the focusing point of the beam, since the amount of energy deposited was above the breakdown threshold for a gaseous mixture.

Given a kernel was initiated by the spark, ignition was strongly associated with the initial kernel size for all conditions with low prevaporisation, suggesting that fluctuations of the overall equivalence ratio at the spark zone controlled the ignition process. This was also supported by the fact that, given a kernel was formed in conditions of higher prevaporisation, ignition was virtually always achieved. Finally, a positive correlation between kernel size and absorbed energy was observed. The dependence of ignition on kernel size and degree of prevaporisation was, therefore, associated to the occurrence of flammable mixture in the spark zone, as increasing both parameters gave rise to smaller fluctuations of fuel in that location.

Timescales of ignition failure were evaluated based on the duration of the overdrive effect of the spark on the flame, $t_{c r i t}$, and timescale statistics were given for a range of conditions. The duration of the overdrive effect on the flame varied from $0.7 \mathrm{~ms}$ for lean conditions and decreased to approximately $0.4 \mathrm{~ms}$ for richer mixtures, being similar to $t_{\text {crit }}$ values found in DNS works. The short mode of ignition failure was characteristic of kernels below a minimum size for ignition $(1 \mathrm{~mm})$, and was also verified in kernels with more than twice the minimum size. Density functions of kernel sizes conditional to ignition or quenching were also presented, offering a way to implement 
part of the stochasticity related to the spark in simulations of ignition of real combustors operating with sprays.

\section{Acknowledgements}

P.M. de Oliveira is grateful for the financial support of the Brazilian Space Agency and Brazil's National Council for Scientific and Technological Development. This work has also been partly funded by the European Commission Clean Sky project AMEL (641453). The authors kindly acknowledge M.P. Sitte and A. Giusti for the helpful discussions, and the assistance of T. Higuchi (Keio University) with the schlieren setup.

\section{References}

[1] E. Mastorakos, Ignition of turbulent non-premixed flames, Prog. Energy Combust. Sci. 35 (2009) 57-97.

[2] N. Chakraborty, E. Mastorakos, R. S. Cant, Effects of turbulence on spark ignition in inhomogeneous mixtures: a direct numerical simulation (DNS) study, Combust. Sci. Technol. 179 (2007) 293-317.

[3] A. D. Birch, D. R. Brown, M. G. Dodson, Ignition probabilities in turbulent mixing flows, Symp. (Int.) Combust. 18 (1981) 1775-1780.

[4] T. X. Phuoc, Laser-induced spark ignition fundamental and applications, Opt. Lasers Eng. 44 (2006) 351-397.

[5] E. Mastorakos, Forced ignition of turbulent spray flames 36 (2017) 2375-2391.

[6] A. M. Danis, I. Namer, N. P. Cernansky, Droplet size and equivalence ratio effects on spark ignition of monodisperse N-heptane and methanol sprays, Combust. Flame 74 (1988) 285-294.

[7] H. El-Rabii, G. Gaborel, J.-P. Lapios, D. Thévenin, J. Rolon, J.-P. Martin, Laser spark ignition of two-phase monodisperse mixtures, Opt. Commun. 256 (2005) 495-506.

[8] A. P. Wandel, N. Chakraborty, E. Mastorakos, Direct numerical simulations of turbulent flame expansion in fine sprays, Proc. Combust. Inst. 32 (2009) 2283-2290. 
[9] A. K. Singh, C. E. Polymeropoulos, Spark ignition of aerosols, Symp. (Int.) Combust. 21 (1988) 513-519.

[10] K. G. Moesl, K. G. Vollmer, T. Sattelmayer, J. Eckstein, H. Kopecek, Experimental Study on Laser-Induced Ignition of Swirl-Stabilized Kerosene Flames, J. Eng. Gas Turbines Power 131 (2009) 021501.

[11] T. Marchione, S. Ahmed, E. Mastorakos, Ignition of turbulent swirling n-heptane spray flames using single and multiple sparks, Combust. Flame 156 (2009) 166-180.

[12] C. Letty, E. Mastorakos, A. R. Masri, M. Juddoo, W. O'Loughlin, Structure of igniting ethanol and n-heptane spray flames with and without swirl, Exp. Therm. Fluid Sci. 43 (2012) 47-54.

[13] D. R. Ballal, A. H. Lefebvre, Ignition and Flame Quenching of Quiescent Fuel Mists, Proc. R. Soc. A Math. Phys. Eng. Sci. 364 (1978) 277-294.

[14] D. R. Ballal, A. H. Lefebvre, Ignition and flame quenching of flowing heterogeneous fuel-air mixtures, Combust. Flame 35 (1979) 155-168.

[15] D. L. Dietrich, N. P. Cernansky, M. B. Somashekara, I. Namer, Spark ignition of a bidisperse, n-decane fuel spray, Symp. (Int.) Combust. 23 (1991) 1383-1389.

[16] E. Mastorakos, Forced ignition of turbulent spray flames, Proc. Combust. Inst. (2016) 1-39.

[17] A. P. Wandel, Influence of scalar dissipation on flame success in turbulent sprays with spark ignition, Combust. Flame 161 (2014) 2579-2600.

[18] J. Kariuki, E. Mastorakos, Experimental investigation of turbulent flames in uniform dispersions of ethanol droplets, Combust. Flame 179 (2017) 95-116.

[19] D. Bradley, C. G. W. Sheppard, I. M. Suardjaja, R. Woolley, Fundamentals of high-energy spark ignition with lasers, Combust. Flame 138 (2004) 55-77.

[20] J. Reveillon, L. Vervisch, Analysis of weakly turbulent dilute-spray flames and spray combustion regimes, J. Fluid Mech. 537 (2005) 317. 
[21] H. Coleman, W. Steele, Experimentation, Validation, and Uncertainty Analysis for Engineers, 3rd ed. ed., John Wiley \& Sons, Inc., Hoboken, 2009.

[22] R. Moffat, Describing the uncertainties in experimental results, Exp. Therm. Fluid Sci. 1 (1988) 3-17.

[23] COSILAB, Cosilab collection, v3.3.2, Rotexo-Softpredict-Cosilab GmbH and Co. KG Bad Zwischenahn (Germany), 2012.

[24] N. M. Marinov, A detailed chemical kinetic model for high temperature ethanol oxidation, Int. J. Chem. Kinet. 31 (1999) 183-220.

[25] T. Poinsot, D. Veynante, Theoretical and numerical combustion, Edwards, Philadelphia, 2001.

[26] A. Neophytou, E. Mastorakos, R. S. Cant, The internal structure of igniting turbulent sprays as revealed by complex chemistry DNS, Combust. Flame 159 (2012) 641-664.

[27] N. Chakraborty, E. Mastorakos, Direct numerical simulations of localised forced ignition in turbulent mixing layers: The effects of mixture fraction and its gradient, Flow, Turbul. Combust. 80 (2008) 155-186.

[28] G. C. Gebel, T. Mosbach, W. Meier, M. Aigner, Optical and spectroscopic diagnostics of laser-induced air breakdown and kerosene spray ignition, Combust. Flame 162 (2015) 1599-1613.

[29] J. L. Beduneau, N. Kawahara, T. Nakayama, E. Tomita, Y. Ikeda, Laserinduced radical generation and evolution to a self-sustaining flame, Combust. Flame 156 (2009) 642-656.

[30] C. Cardin, B. Renou, G. Cabot, A. M. Boukhalfa, Experimental analysis of laser-induced spark ignition of lean turbulent premixed flames: New insight into ignition transition, Combust. Flame 160 (2013) 1414-1427.

[31] Y. Hardalupas, M. Orain, Local measurements of the time-dependent heat release rate and equivalence ratio using chemiluminescent emission from a flame, Combust. Flame 139 (2004) 188-207.

[32] M. Rosenblatt, Remarks on Some Nonparametric Estimates of a Density Function, Ann. Math. Stat. 27 (1956) 832-837. 
[33] E. Parzen, On Estimation of a Probability Density Function and Mode, Ann. Math. Stat. 33 (1962) 1065-1076.

[34] D. B. Spalding, Combustion and mass transfer: a textbook with multiple-choice exercises for engineering students, Pergamon Press, 1979.

[35] A. Neophytou, E. Mastorakos, R. S. Cant, DNS of spark ignition and edge flame propagation in turbulent droplet-laden mixing layers, Combust. Flame 157 (2010) 1071-1086.

[36] A. Müsing, U. Riedel, J. Warnatz, W. Herden, H. Ridderbusch, Laserinduced breakdown in air and behind droplets: A detailed Monte-Carlo simulation, Proc. Combust. Inst. 31 II (2007) 3007-3014.

[37] G. C. Gebel, T. Mosbach, W. Meier, M. Aigner, Laser-induced blast waves in air and their effect on monodisperse droplet chains of ethanol and kerosene, Shock Waves 25 (2015) 415-429.

\section{Appendix A - Flow characteristics}

A summary of the results of flow measurements to characterise the flow at the burner exit is presented.

\section{A.1 Droplet size}

The uniformity of droplet sizes across the jet for the range of equivalence ratios investigated is represented in Fig. 16. Nine curves are shown in each plot, concerning pdfs of size measurements taken every $2 \mathrm{~mm}$ along the radial coordinate of the jet, starting at $2 \mathrm{~mm}$ from the mixing layer of the jet. The spatial-averaged Sauter mean diameter $\left\langle d_{32}\right\rangle$ is shown, with the grey area indicating the variation of this parameter across the jet. Some variation of $d_{32}$ in terms of $r$ was mainly seen in experiments with high equivalence ratios, being related to a small variation of the number distribution at large droplet sizes, therefore resulting in a significant change of $d_{32}$ for the whole distribution.

Typical number and volume distribution based on the droplet size are shown in Fig. 17. It should be noted that a significant portion of the total fuel lies in a small number of large droplets which are scarce in the flow. Thus, 

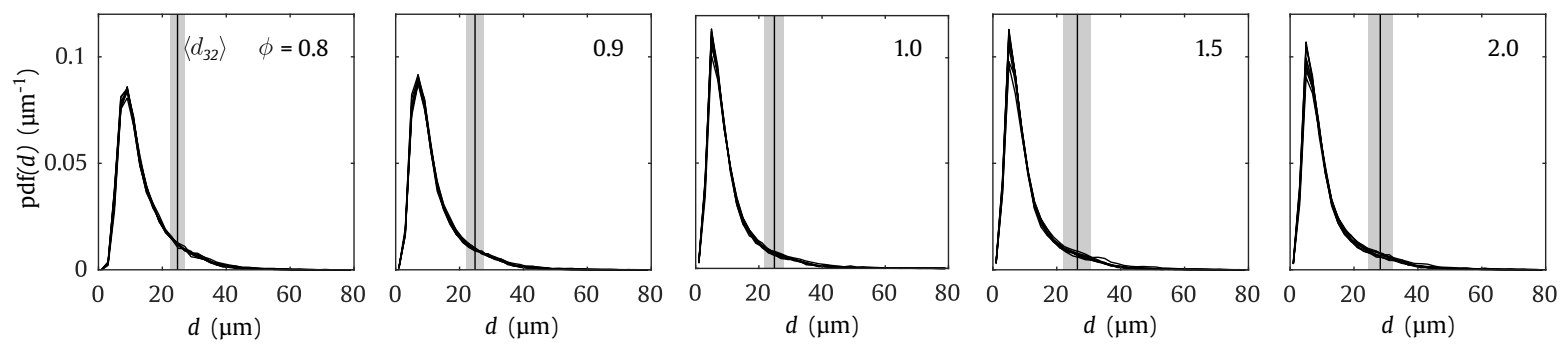

(a) $30^{\circ} \mathrm{C}$
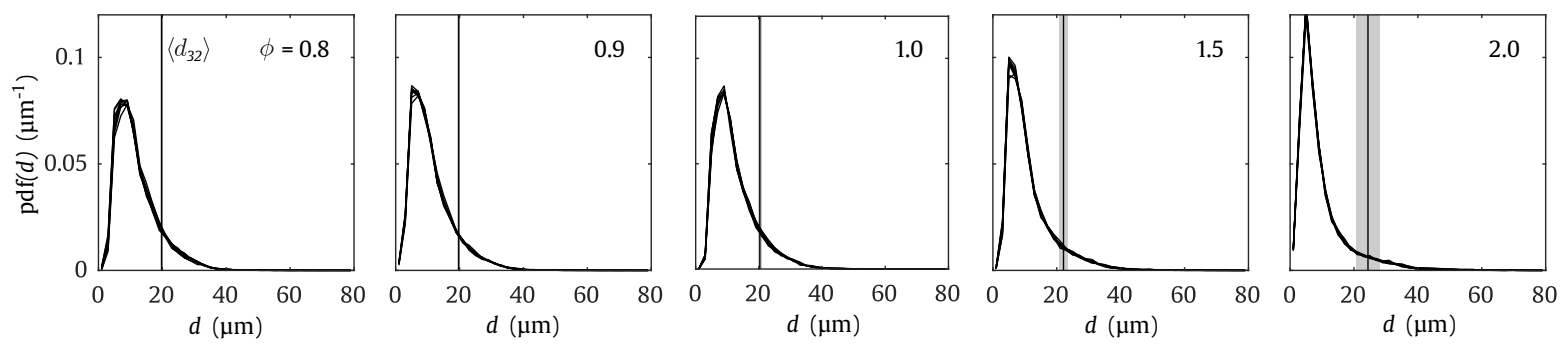

(b) $50^{\circ} \mathrm{C}$

Figure 16: Probability density functions of droplet size for different preheat conditions: (a) $T_{i n}=30^{\circ} \mathrm{C}$ and (b) $50^{\circ} \mathrm{C}-U_{b}=6 \mathrm{~m} / \mathrm{s}$. For each equivalence ratio, 9 overlapping pdfs concerning different radial positions are shown.

the droplet size distributions were expressed in terms of Rosin-Rammler and Modified Rosin-Rammler distributions, which allows for a precise implementation of the spray characteristics in future simulations of the present experiment. The distributions are given in terms of the accumulated percent volume $Q$,

$$
Q(k)=\frac{\sum_{i=1}^{k} N_{i} d_{i}^{3}}{\sum_{i=1}^{\infty} N_{i} d_{i}^{3}} .
$$

The Rosin-Rammler distribution is then given by,

$$
Q(d)=1-\exp \left[-\left(\frac{d}{X}\right)^{q}\right],
$$

and its modified form,

$$
Q(d)=1-\exp \left[-\left(\frac{\ln d}{\ln X_{m}}\right)^{q_{m}}\right] .
$$




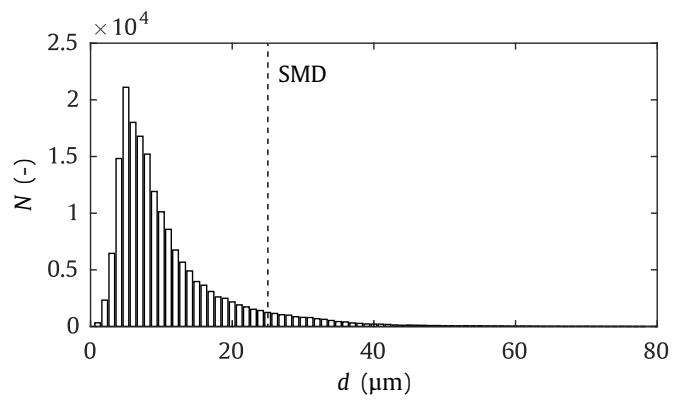

(a)

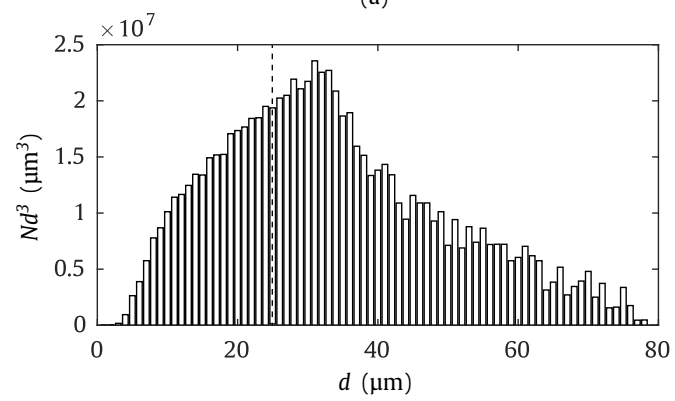

(b)

Figure 17: Typical histograms of (a) droplet size and (b) liquid volume distribution $\left(\sim N d^{3}\right)$ concerning all measurements taken within the uniform part of the jet $-T_{i n}=$ $30^{\circ} \mathrm{C}, U_{b}=6 \mathrm{~m} / \mathrm{s}$, and $\phi=1$.

The parameters $X$ and $q$ of the regular and the modified distributions were obtained by fitting of the experimental data. A characteristic Rosin-Rammler plot showing both distributions for a specific test condition is shown in Fig. 18. All experimental conditions followed the modified form of the RosinRammler distribution, as seen in Fig. 18b. The distributions for the experiments given in Table 2 are given in Table 3 .

\section{A.2 Velocity}

The end velocity of the droplets at the exit of the nozzle is illustrated in Fig. 19a. Mean axial velocity and turbulence are shown, and were calculated according five classes, $d(\mu \mathrm{m})$, of droplet size: $d<5 ; 5<d<10 ; 10<d<30$; $30<d<50 ; d>50$.

Overall, for the range of conditions investigated, about $95 \%$ of the total number of droplets were within the first 3 categories, that is, are smaller than $30 \mu \mathrm{m}$. Figure 19a shows that the mean axial velocity of these small droplets are virtually the same across the profile, therefore, no drift between 


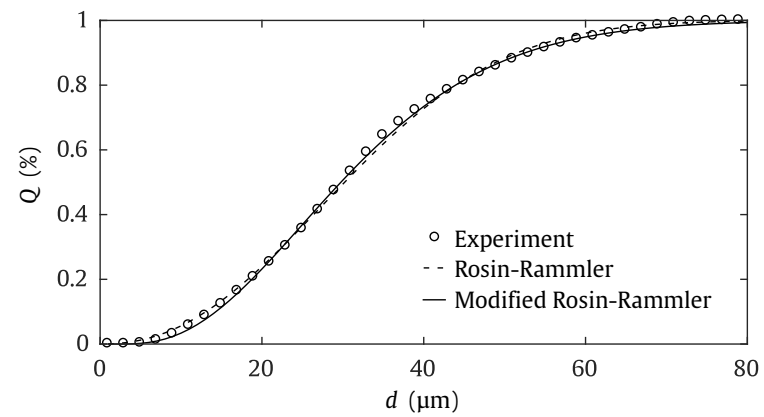

(a)

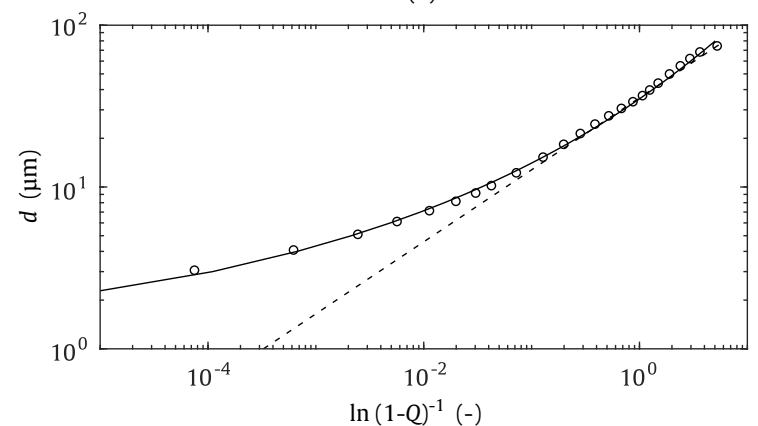

(b)

Figure 18: (a) Accumulated volume in terms of droplet size and (b) typical Rosin-Rammler plot $-T_{\text {in }}=30^{\circ} \mathrm{C}, U_{b}=6 \mathrm{~m} / \mathrm{s}$, and $\phi=0.9$.

the gas and the liquid phase existed. However, droplets larger than $30 \mu \mathrm{m}$ were found to be slower, with axial velocity of -0.3 and $-0.9 \mathrm{~m} / \mathrm{s}$ relative to the gas phase, for classes $30<d<50$ and $d>50$, respectively. The largest droplets represented approximately $10 \%$ of the fuel, and their relative velocity may have affected the growth of the flame. Relative to the flow, large droplets moved towards the upper flame front and away from the lower front as the spherical flame expanded. The profile of the rms turbulent velocity for each droplet class is shown in Figure 19b, showing a clear effect of the mixing layer with a thickness of approximately $2 \mathrm{~mm}$. Droplets of up to $10 \mu \mathrm{m}$ were found to be equally responsive to velocity fluctuations.

The velocity of the gas phase was determined from droplets smaller than $10 \mu \mathrm{m}$. From that, the flow in the region of interest was evaluated in order to verify the limits in which the uniformity of the flow applied. Figure 20 shows radial profiles of the mean velocity and the rms velocity fluctuation of the gas phase for three axial positions $z / d$ within the first diameter of the jet $\left(\bar{U}_{b}=15 \mathrm{~m} / \mathrm{s}, \phi=0.1\right)$. A uniform top-hat velocity profile was verified at the 
Table 3: Parameters of the Rosin-Rammler and the Modified Rosin-Rammler distributions.

\begin{tabular}{c|cccc}
\hline$\#$ & $X$ & $q$ & $X_{m}$ & $q_{m}$ \\
\hline 1 & 34.5 & 2.31 & 34.0 & 7.88 \\
2 & 35.6 & 2.25 & 35.1 & 7.76 \\
3 & 37.3 & 2.13 & 36.7 & 7.45 \\
4 & 39.2 & 2.22 & 38.7 & 7.90 \\
5 & 41.6 & 2.26 & 41.0 & 8.18 \\
\hline 6 & 26.1 & 2.63 & 25.8 & 8.22 \\
7 & 26.5 & 2.49 & 26.2 & 7.81 \\
8 & 27.0 & 2.49 & 26.7 & 7.83 \\
9 & 31.3 & 2.31 & 30.8 & 7.63 \\
10 & 36.7 & 2.21 & 36.2 & 7.71 \\
\hline \hline 11 & 44.1 & 2.29 & 43.5 & 8.34 \\
12 & 44.5 & 2.34 & 43.9 & 8.58 \\
\hline 13 & 39.7 & 2.11 & 39.0 & 7.47 \\
14 & 43.5 & 2.10 & 42.9 & 7.60
\end{tabular}

exit of the burner, and the thickness of the mixing layer can be noticed in the velocity profiles, increasing as $z / d$ increases. This region was determined, and measurements of ignition and flame growth in the experiments were limited to the region where the profile is uniform (Fig. 20).

Additionally, the mean axial velocity and turbulence were also assessed across the profile for the range of conditions investigated (Fig. 21). The variation of the mean velocity and rms velocity fluctuation along the radial coordinate is shown as an error-bar for each marker. Experiments were carried out in a way such the centreline velocity was kept constant. Nevertheless, $\left\langle\bar{u}_{z}\right\rangle$ varied within $\pm 3 \%$ due to changes in the carrier and atomising flow rates as $\phi$ changed, and also due to evaporation of the liquid fuel which also affects the flow rate and temperature of the gas phase.

\section{A.3 Fuel prevaporisation}

The amount of fuel prevaporised upstream the region of interest, $\dot{m}_{f, g}$, was estimated from an energy balance of the flow in the burner,

$$
\left[\dot{m}_{l} h_{l}\left(T_{l}\right)+\dot{m}_{g} h_{g}\left(T_{g}\right)\right]_{\text {in }}=\left[\dot{m}_{l} h_{l}\left(T_{l}\right)+\dot{m}_{g} h_{g}\left(T_{g}\right)+\dot{m}_{f, g} h_{l v, f}\right]_{\text {out }}
$$

where the subindices $l$ and $g$ are the liquid and gas phase, respectively, $h_{k}$ is the enthalpy of the given phase $k$ at temperature $T_{k}$, and $h_{l v, f}$ is the enthalpy 


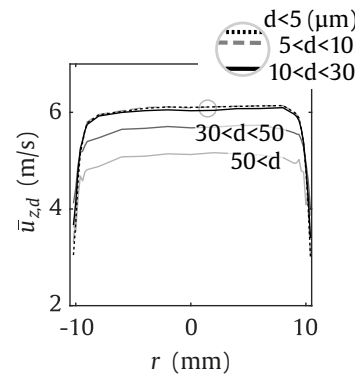

(a)

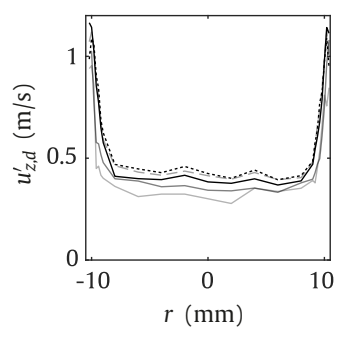

(b)

Figure 19: Radial profiles of (a) axial velocity and (b) turbulent fluctuation according to range of droplet sizes $-z / d=0.2, U_{b}=6 \mathrm{~m} / \mathrm{s}$, and $\phi=1$.
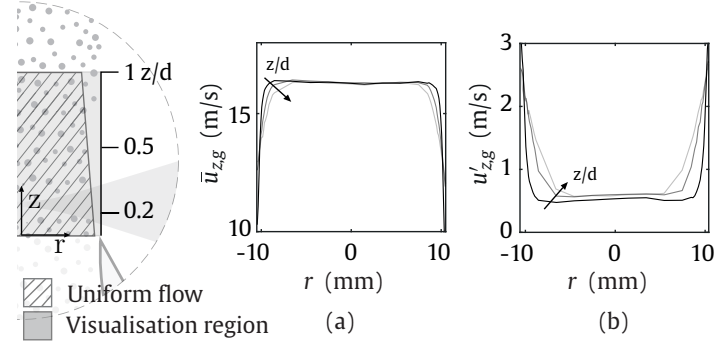

Figure 20: Radial profiles of (a) axial velocity and (b) turbulent fluctuation, taken along the axial coordinate of the jet within the region of interest at $z / d$ of $0.2,0.5$, and $1-$ $U_{b}=15 \mathrm{~m} / \mathrm{s}$ and $\phi=0.1$.

of vaporisation of the fuel. At the inlet, $\dot{m}_{g}$ is simply the total measured mass flow rate of air, $T_{l}$ is taken as the ambient temperature, and $T_{g}$ is the preheating temperature of the air measured upstream of the atomiser. At the outlet, the gas phase mass flow rate is,

$$
\left.\dot{m}_{g}\right|_{\text {out }}=\left.\dot{m}_{g}\right|_{\text {in }}+\left.\dot{m}_{f, g}\right|_{\text {out }} \text {. }
$$

Given that the residence time of the droplets inside the burner was verified to be relatively long in Large Eddy Simulations of the present experiment (M.P. Sitte, personal communication), saturation of fuel in the gas phase was assumed at the outlet of the nozzle, as well as thermal equilibrium of the gas and liquid phases $\left(T_{g}=T_{l}\right)$. The total amount of fuel vaporised is given by,

$$
\left.\dot{m}_{f, g}\right|_{\text {out }}=\left.y_{f, g}^{\text {sat }} \dot{m}_{g}\right|_{\text {out }},
$$



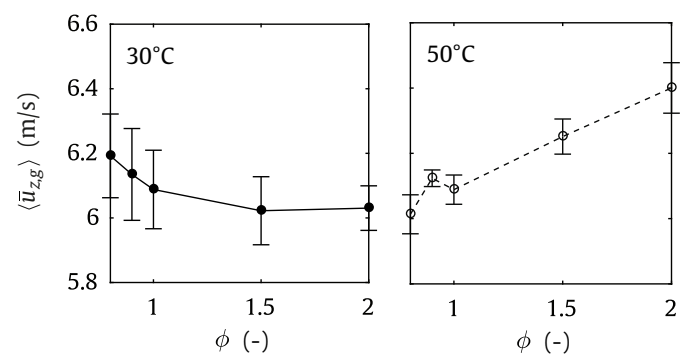

(a)
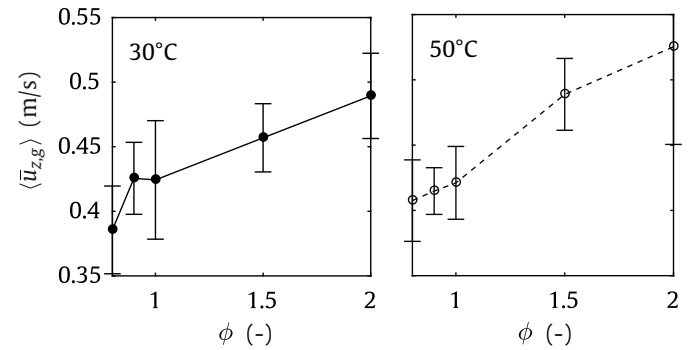

(b)

Figure 21: The (a) velocities and (b) turbulent fluctuations in the axial direction concerning the uniform part of the top-hat velocity profile for 30 and $50^{\circ} \mathrm{C}-U_{b}=6 \mathrm{~m} / \mathrm{s}$. Error bars represent the variations along the radial coordinate.

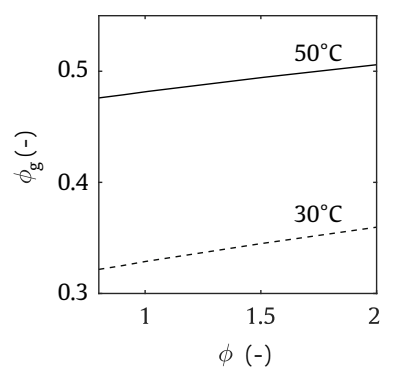

Figure 22: Calculated equivalence ratio of the gas phase in terms of the overall equivalence ratio.

where the mass fraction of fuel for saturation is,

$$
y_{f, g}^{s a t}=\frac{x_{f, g}^{s a t} M W_{f}}{\left(x_{f, g}^{s a t} M W_{f}+\left(1-x_{f, g}^{s a t}\right) M W_{a i r}\right)},
$$

and $x_{f, g}^{s a t}$ depends on the saturation pressure of the fuel at the gas-phase 
temperature, and is evaluated from its partial pressure,

$$
x_{f, g}=\frac{P_{s a t, f}\left(T_{g}\right)}{P_{a t m}} .
$$

The enthalpy of the gas mixture in Eq. (6) was evaluated by taking the specific heat capacity of the mixture assuming an ideal mixture. Equations (6-10) were solved numerically using thermodynamic tables for $h$ and $c_{p}$ of air and ethanol. Figure 22 shows the calculated equivalence ratio of the gas phase, $\phi_{g}$. A mean value of 0.34 and 0.49 was obtained for preheating temperatures of the air flow upstream the atomiser of $30^{\circ} \mathrm{C}$ and $50^{\circ} \mathrm{C}$, respectively. 